University of Zurich

Department of Economics

Working Paper Series

ISSN 1664-7041 (print)

ISSN 1664-705X (online)

Working Paper No. 334

\title{
Timing of Predictions in Dynamic Cheap Talk: Experts vs. Quacks
}

Aleksei Smirnov and Egor Starkov

November 2019 


\title{
Timing of Predictions in Dynamic Cheap Talk: Experts vs. Quacks*
}

\author{
Aleksei Smirnov ${ }^{\dagger}$ Egor Starkov $\ddagger$
}

November 11, 2019

\begin{abstract}
The paper studies a dynamic communication game in the presence of adverse selection and career concerns. A forecaster of privately known competence, who cares about his reputation, chooses the timing of the forecast regarding the outcome of some future event. We find that in all equilibria in a sufficiently general class earlier reports are more credible. Further, any report hurts the forecaster's reputation in the short run, with later reports incurring larger penalties. The reputation of a silent forecaster, on the other hand, gradually improves over time.
\end{abstract}

Keywords: Career concerns, reputation, dynamic games, games of timing, strategic information transmission.

JEL Codes: C73, D82, D83, D84.

\footnotetext{
${ }^{*}$ We are grateful to Antonio Cabrales, Vincent Crawford, Jeffrey Ely, Yingni Guo, Stephen Morris, Nick Netzer, Wojciech Olszewski, Marek Pycia, Debraj Ray, Armin Schmutzler, Joel Sobel, Bruno Strulovici, seminar participants at Northwestern University, University of Zürich, NHH Bergen and Helsinki GSE, as well as participants of 2017 OLIGO Workshop, the 9th edition of The Lisbon Meetings in Game Theory and Applications and 2019 Econometric Society Summer School for valuable feedback and helpful comments.

${ }^{\dagger}$ Department of Economics, University of Zürich, Blümlisalpstrasse 10, CH-8006, Zürich, Switzerland; e-mail: aleksei.smirnov@econ.uzh.ch

${ }^{\dagger}$ Department of Economics, University of Copenhagen, Øster Farimagsgade 5, bygning 26, 1353 København K, Denmark; e-mail: egor@starkov.email
} 


\section{Introduction}

Where there is uncertainty, there are analysts - be it stock prices, macroeconomic trends, elections, or sports matches. Any major event summons numerous predictions of its outcome from people who claim to be experts in the field. However, not all of these predictions are necessarily backed by knowledge or understanding of the situation. This raises challenges for both sides of the predictions market. The expert forecasters must find a way to signal their competence through their predictions. The public, on the other hand, must find a way to identify informative predictions by the experts among the quacks' uninformative opinions.

This paper focuses on the timing of the forecaster's prediction as a signaling device. We explore the questions of how the choice of timing of the forecaster's prediction can signal their competence and how the amount of information about the state of the world contained in the predictions changes over time. To motivate the question, consider the case of 2016 US President Elections. Figure 1 demonstrates the ratings of presidential candidates Donald Trump and Hillary Clinton in a span of 9 month before the elections. Consider three different claims that Donald Trump will win, made in February 2016 (before Republican Party presidential primaries), May 2016, and September 2016 respectively 11 Which of these predictions appears more credible ex post? Which of these predictions appeared more credible at the time? Would the authors of the early predictions have benefited from delaying it and, if yes, why did they not? Would the authors of the later predictions have made their predictions earlier if they had the information? These are the questions we attempt to answer in this paper.

We present a model of dynamic cheap talk with career concerns. In our model a forecaster, who is privately aware of his competence, makes a choice of whether and when to make a prediction about the outcome of some future exogenous event (state of the world). A competent forecaster (an expert) may have some private knowledge about the outcome, while an incompetent forecaster (a quack) never does. There is no direct conflict between the forecaster and the observer (the public) in our model: the forecaster only cares about his reputation, while the observer only cares about the information concerning the outcome. The conflict comes from within the forecasters market, with the quacks trying to blend in with the experts in pursuit of reputation (and benefits that high reputation grants), preventing experts from conveying valuable information to the public.

We discover that this conflict between the quacks and the experts imposes a lot of structure on equilibrium outcomes. Our first finding is that in equilibrium, the later predictions are less informative than the earlier ones. Otherwise - if later predictions are more informative - they are also rewarded with higher reputation by the public, which would make the experts want to delay their reports. Therefore, the informativeness of predictions must deteriorate over time to incentivize the experts to reveal their information early, and to prevent a market shutdown when no predictions are made until the very final moment.

A more surprising finding of our paper is that all predictions in such equilibria, although

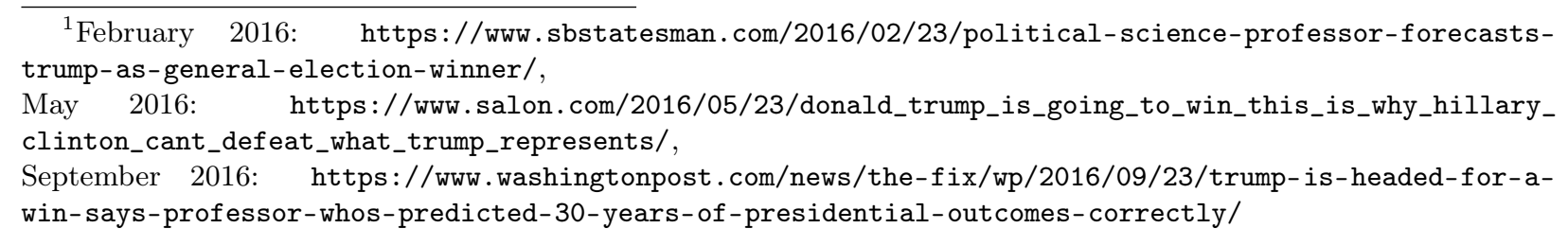




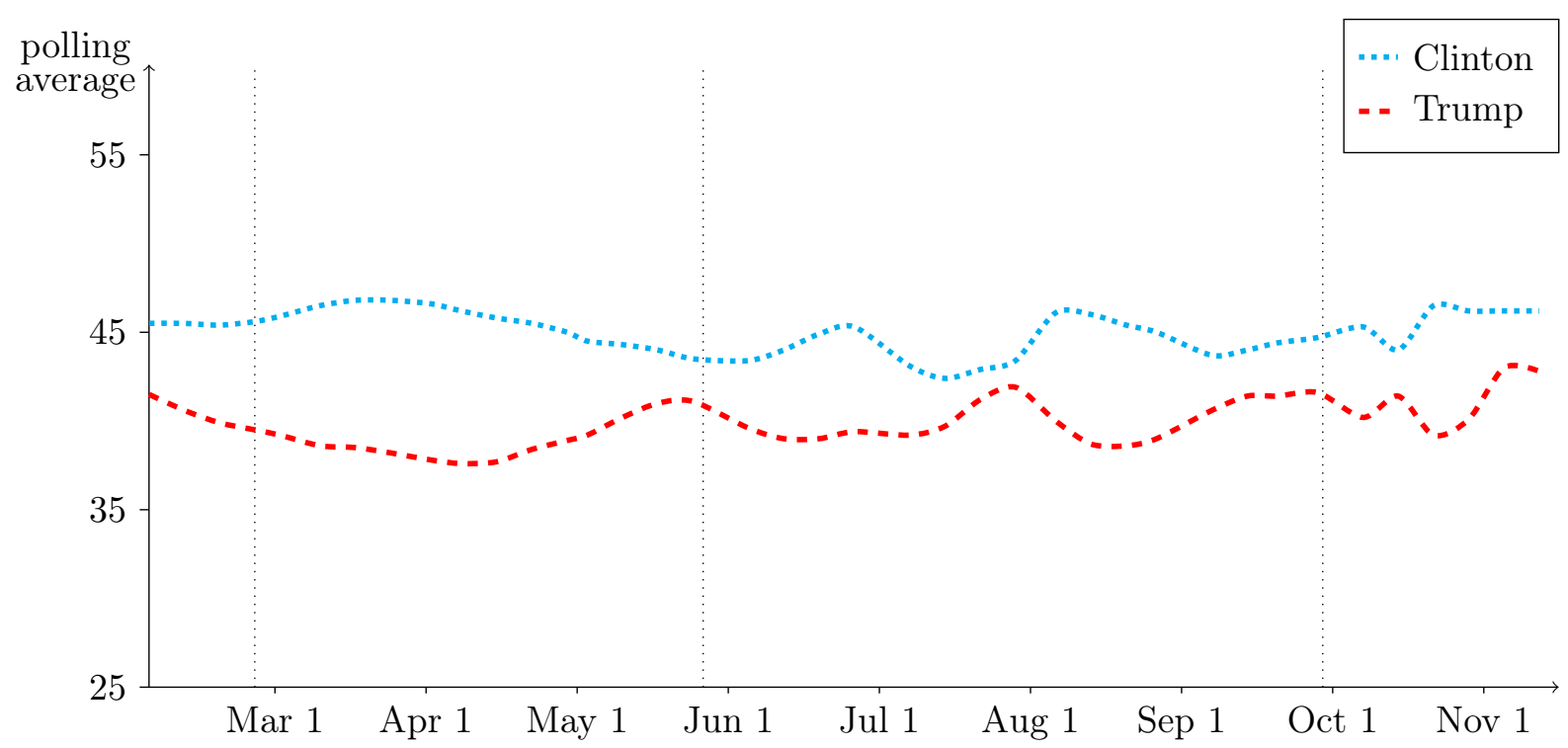

Figure 1: Donald Trump and Hillary Clinton polling averages before the 2016 elections. Dotted verticals represent the dates of news articles mentioned in the text. Poll data retrieved from https:// elections. huffingtonpost. com/ pollster/2016-general-election-trump-vs-clinton, used under CC 3.0 BY-NC-SA license.

considered informative, are received with solid scepticism by the public. This is in the sense that making any prediction drops the forecaster's reputation relative to what he could get by staying quiet. Thus silence is indeed golden in our model - silent forecasters see their reputation gradually improving. Those, on the other hand, who choose to make a prediction and take a hit to their reputation, are gambling for the grand prize that is the reputation bonus for predicting the outcome correctly.

A typical path of the forecaster's reputation arising from our model is illustrated in Figure 2. In this example the event occurs in period 6 and the forecaster starts with reputation $b_{0}$. The forecaster makes his report in period 4, and until then his reputation gradually increases. After the report his reputation drops until the event outcome is revealed, at which point he receives a reputation premium if his prediction turned out correct and is penalized by low reputation otherwise.

Given everything said above, it is not obvious why a forecaster would ever prefer to make any prediction, i.e., take a risky gamble at the cost of short-run reputation, when staying silent would yield a risk-free high reputation. As we show, equilibria of the form above only exist if forecasters are sufficiently risk-loving or, alternatively, if gains from reputation are sufficiently convex - i.e., if the gamble of making a report is appealing enough to the quack. Whenever this is not the case, only "static" equilibria exist, in which all reports are made at some single predetermined date $2^{2}$

The paper is organized as follows. Section 2 contains a review of the relevant literature. Section 3 presents our results in the simplest setting. In Section 4 we formulate the general model. The main results are presented in Section 5. Section 6 contains extensions and alternative specifications. Section 7 concludes. All proofs are relegated to the Appendix.

\footnotetext{
${ }^{2}$ Under special assumptions there also exist degenerate equilibria, in which quack never makes any predictions for the fear of being proved wrong. See Section 6.3 for details.
} 


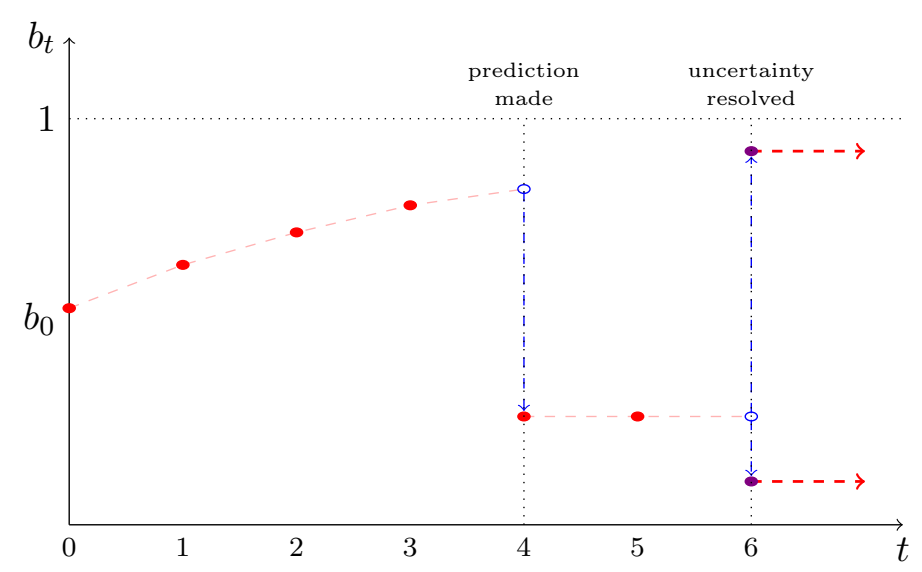

Figure 2: Example forecaster's reputation path.

\section{Relation to the Literature}

The current paper mainly contributes to two strands of literature: communication with career concerns and the timing of communication.

The importance of career concerns for informative communication was first argued by Holmström [1999]. One of Holmström's original examples illustrates that an expert may be reluctant to truthfully reveal his private information for fear of making a mistake and appearing incompetent, preferring instead to herd with public information or reports of other experts 3 Other papers have argued that some cohort of analysts - or even all of them in some settings - may, conversely, resort to extreme reports, overstating their private signals in order to separate themselves from "herders" (see Prendergast and Stole [1996, Graham 1999], Hong, Kubik, and Solomon 2000, Lamont 2002, Ottaviani and Sørensen 2006b, Mariano 2012]). Either way, it is generally agreed that experts'

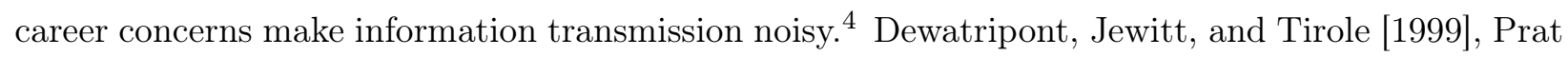
2005, Ottaviani and Sørensen 2006c, and Rodina 2017 give various general characterizations of communication outcomes in the presence of career concerns and their dependence on the information structure of the game.

Of all papers mentioned above only a few look at the dynamics of announcements. In the model of Prendergast and Stole [1996] the expert obtains his private information gradually over time, and his competence determines the speed of learning. They establish that the experts overreact to early pieces of information in order to establish their reputation for competency early on, while as time progresses, they become too reluctant to change their decisions and thus underreact to late information. Predictions of a model by Graham [1999] can be interpreted in a similar way ${ }^{5}$ Hong, Kubik, and Solomon 2000 and Lamont 2002 find a completely opposite pattern in the data: as the experts become older and more established, they usually make more extreme predictions. However, timing of the prediction or a decision is never a choice variable for the expert in these papers. Our

\footnotetext{
${ }^{3}$ This idea was picked up and greatly extended upon by the literature that followed: see Scharfstein and Stein 1990, Trueman 1994, Ely and Välimäki 2003], Ottaviani and Sørensen 2006a], Dasgupta and Prat [2008.

"Zábojnik 2001, Ely and Välimäki 2003 and Klein and Mylovanov|2017 argue that if all experts are patient enough then this noise vanishes and communication efficiency is restored. Backus and Little 2018] show that making experts admit uncertainty (not knowing the answer) is also not a trivial problem in the presence of career concerns.

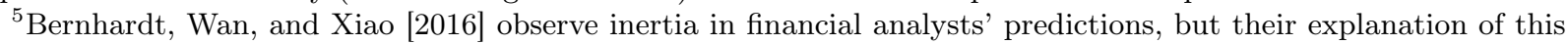
phenomenon does not rely on career concerns.
} 
paper fills this gap by examining how an expert can manipulate his reputation by strategically choosing the timing of his prediction.

Keskek, Tse, and Tucker 2014 provide evidence from the field that competent experts tend to make their reports earlier - so earlier reports are more informative and are perceived more favorably, - and explain this through preemption mechanisms. We show that competition is not necessary for this phenomenon to arise.

The second large (and growing) strand of literature this paper contributes to is that on dynamic communication and, especially, the timing of communication 6 Guttman, Kremer, and Skrzypacz [2014] provide a notable illustration on the importance of timing in communication. In the context of dynamic disclosure, they show that the same piece of hard (verifiable) information can induce different reactions when disclosed at different times. In other related papers, Guttman [2010], Acharya, DeMarzo, and Kremer 2011, Aghamolla and An 2015, and Gratton, Holden, and Kolotilin [2017] also investigate optimal timing in the context of dynamic disclosure of verifiable information. In contrast to these papers, we deal with soft information, which cannot be credibly disclosed. Grenadier, Malenko, and Malenko [2016] study a setting in which the informed expert uses timing of his (non-verifiable) report to manipulate the timing of the observer's decision. A separate literature explores dynamic revelation of static information and finds, to some surprise, that even if all agents possess all of their respective information in period zero, allowing for multiperiod communication may sometimes allow for higher payoffs to some or all parties.7 All of the aforementioned communication models assume direct conflict of interest between the sender(s) and the receiver(s). Our model of career concerns is different in this regard, since all barriers to truthful communication stem instead from the conflict within the senders' market, namely between competent and incompetent forecasters ${ }^{8}$

Finally, our paper takes the market for predictions as given rather than designing it in such a way as to extract the most information from the expert. A general approach to dynamic mechanism design when experts have evolving private information has been proposed by Pavan, Segal, and Toikka 2014. A sub-field of mechanism design explicitly deals with optimal statistical testing of experts' competence (knowledge of a signal-generating process): see Olszewski [2015] for a recent survey ${ }^{9}$ Our paper is different from this literature in that it does not give the observer the power to design payoffs or information feedback. Instead, it asks the question of whether market forces alone can enable informative communication.

\section{Illustrative Example}

This section presents an example that showcases our main results in the simplest setting. Suppose there are two periods $t=1,2$ and a binary state $\omega \in\{G, B\}$, which is initially not

\footnotetext{
${ }^{6}$ For dynamic models of repeated communication, where the sender does not have the choice of timing, see Sobel 1985, Bénabou and Laroque 1992, Morris 2001, Pavesi and Scotti 2017.

'See Aumann and Hart 2003, Krishna and Morgan 2004, Alonso and Rantakari 2013, Chen, Goltsman, Hörner, and Pavlov [2017], and Lipnowski and Ravid [2019].

${ }^{\circ}$ Curiously, effects similar to career concerns models can be obtained in communication settings with senderreceiver conflict where the sender's deceit can be detected with positive probability. For examples of such models see Dziuda and Salas 2019 and Drugov and Troya-Martinez 2019].

${ }^{9}$ The most recent contributions to this field include Di Pei 2016, Ginzburg 2019, Smolin 2018, and Deb, Pai, and Said 2018.
} 
known to anybody and is publicly revealed in the end of period $T=2$. Assume that players do not discount the future, and that states are ex ante equally probable, i.e. $\operatorname{Pr}(\omega=G)=\operatorname{Pr}(\omega=B)=\frac{1}{2}$.

There are two players: a forecaster and an observer. The forecaster is, with equal probabilities, either an expert, or a quack. The forecaster privately knows his type, but the observer does not. The expert has a chance $\lambda_{t}$ to privately learn the state in period $t=1,2$. With positive probability, the expert also remains unaware of the state, i.e., $\lambda_{1}+\lambda_{2}<1$. The quack never receives any private information about the state. In any of the two periods before the state is publicly revealed, the forecaster can send one cheap talk report $m \in\{G, B\}$ to the observer, indicating his prediction about state $\omega$. The report is not verifiable, i.e., the forecaster's private information can not be made observable to the public. At the end of each period the forecaster receives a "reputation payoff" equal to the probability that the observer assigns at that moment to the forecaster being an expert.

We will look for an equilibrium in which the expert is honest: he reports according to his private information as soon as he obtains it and never makes an unfounded prediction or reports contrary to his information (this behavior will be optimal in equilibrium). How would the quack behave in such equilibrium, and how should the market react to either report and to a lack thereof?

There are five actions available to the forecaster in the game: he can report that the state is $G$ or $B$ at $t=1,2$ or stay silent throughout. An honest expert plays all five actions with positive probability. It is immediate then that the quack must do the same in equilibrium - if either action is only taken by the expert and never by the quack, then following this path gives the forecaster the highest possible reputation from that point onwards and, therefore, the highest possible continuation payoff. This would strictly dominate any alternative path of play available to the quack at the respective period.

Therefore, the quack must be indifferent, in particular, between reporting that the state is $G$ at $t=1$ and $t=210$ Denote by $b_{t}$ the belief about the forecaster's competence at the end of period $t$ in case no report was made in period $t$; by $b(m, t)$ the belief after report $m$ at period $t$ was made; and by $b^{\omega}(m, t)$ the belief after report $m$ at period $t$ was made, and the state turned out to be $\omega$. The indifference condition between the two reports for the quack is then given by

$$
b(G, 1)+\left[\frac{1}{2} \cdot b^{G}(G, 1)+\frac{1}{2} \cdot b^{B}(G, 1)\right]=b_{1}+\left[\frac{1}{2} \cdot b^{G}(G, 2)+\frac{1}{2} \cdot b^{B}(G, 2)\right] .
$$

Note that the honest expert is never wrong, since he only makes a report if he knows the state. Therefore, if the forecaster made a prediction which turned out to be incorrect, he is definitely a quack: $b^{B}(G, 1)=b^{B}(G, 2)=0$.

We have assumed that the expert always reveals his information at $t=1$ if he has it. However, he does have an option to delay his report until the second period if he already knows the state at $t=1$. To ensure that there is no delay, the following has to hold:

$$
b(G, 1)+b^{G}(G, 1) \geqslant b_{1}+b^{G}(G, 2) .
$$

Note that the expert's expected utility only differs from that of the quack in the probability of guessing the state correctly - the expert knows that his private signal is correct. The two expressions (1) and (2) together produce our main results described below.

\footnotetext{
${ }^{10}$ We will use message $m=G$ to illustrate our results, but all arguments apply equally to either message.
} 
Early correct reports are rewarded higher ex post. Subtracting equality (1) from (2), we immediately obtain that $b^{G}(G, 1) \geqslant b^{G}(G, 2)$. Early reports must thus be rewarded with higher reputation to incentivize the expert to reveal his information in a timely manner. Note that this only applies to reputation after the state was revealed.

Reporting harms reputation. Combining (1) and (2), we also infer that $b(G, 1) \leqslant b_{1}$, and by analogy we can obtain $b(B, 1) \leqslant b_{1}$. Therefore, any report at $t=1$ must be worse than not making a report.

Reputation of a silent expert improves. By the martingale property of beliefs, we note that $b(G, 1), b(B, 1)$, and $b_{1}$ must average out to $b_{0}$. The inequalities we just obtained then imply that $b_{1} \geqslant b_{0}$ : if reporting harms reputation then staying silent must improve it. With slightly more work, one can also show that $b_{2} \geqslant b_{1} 11$

Early reports are more precise. The previous observations almost immediately imply that earlier reports contain more information about the state. Indeed, $b^{G}(G, 1) \geqslant b^{G}(G, 2)$ and $b^{B}(G, 1)=b^{B}(G, 2)=0$, therefore $b(G, 1) \geqslant b(G, 2)$, since by the martingale property of beliefs, $b^{G}(m, t)$ and $b^{B}(m, t)$ should average out (from the observer's perspective) to $b(m, t)$. Because $b_{1} \geqslant b_{0}$ the latter inequality means that the earlier of the two reports is relatively more likely to be made by the expert - which immediately implies that it is more informative than the latter one.

The following sections expand on the analysis of this example in a general framework and show that the insights demonstrated above are quite general.

\section{The Model}

\subsection{Primitives}

Time is discrete and finite: $t \in\{0\} \cup \mathcal{T}$ where $\mathcal{T} \equiv\{1, \ldots, T\}$ for some $T>0$. An underlying standard probability space is implied throughout the paper. The probability measure on this space is denoted by $P$.

State of the world. There is a binary state of the world $\omega$ which can be either good or bad: $\omega \in\{G, B\}$. The commonly held prior belief that the state is good is $P(\omega=G)=p_{0} \in\left[\frac{1}{2}, 1\right)$. Initially the state is uncertain; at the end of period $T$ the state is revealed.

Players. There are two players: an observer (she) and a forecaster (he). Both players live for $T$ periods and do not discount the future.

The forecaster has a binary type $\gamma \in\{E, Q\}$ : he can be competent or incompetent or, as we call them, an expert (E) or a quack (Q) respectively. The type is privately known by the forecaster, but is not known by the observer. The observer's initial belief that the forecaster is competent is $b_{0} \in(0,1)$.

\footnotetext{
${ }^{11}$ By the martingale property, $b(G, 2), b(B, 2)$, and $b_{2}$ must average out to $b_{1}$, and we know that $b(m, 2) \leqslant b(m, 1) \leqslant$ $b_{1}$ for $m=G, B$. Therefore, $b_{2} \geqslant b_{1}$.
} 
The observer has no actions in the model ${ }^{12}$ In every period $t$ she updates her beliefs $p_{t}$ about the state of the world and $b_{t}$ about the forecaster's competence. It will prove convenient to represent these beliefs as likelihoods rather than probabilities, so let $\rho_{t}=\frac{p_{t}}{1-p_{t}}$ and $\beta_{t}=\frac{b_{t}}{1-b_{t}}$.

At some random time $t^{*} \sim F(t)$, which is not known to anybody, the competent forecaster observes a signal $\eta^{*} \in\{G, B\}$ about the state, with precision $\pi:=P\left(\eta^{*}=G \mid \omega=G\right)=P\left(\eta^{*}=\right.$ $B \mid \omega=B)$. For most of the paper we assume $\pi=1$, but in Section 6.4 we show that all results continue to hold in case $\pi \in\left(\frac{1}{2}, 1\right)$ given some extra conditions. We assume that $F(t)$ is a measure with full support on $\mathcal{T}$ and that $F(T)<1$, i.e., there is a positive probability that the signal arrives at any time $t$ and it is possible that it never arrives. We denote the conditional probability (hazard rate) of signal arrival in period $t$ as $\lambda(t):=\frac{F(t)-F(t-1)}{1-F(t-1)}$.

The forecaster receives a per-period "reputation payoff" $w\left(\beta_{t}\right)$ which depends on the observer's belief about the forecaster's competence held at the end of period $t$. We assume $w(\cdot)$ to be strictly increasing in its argument. As a normalization, we let $w(0)=0$. After the state is revealed, the forecaster receives a terminal payoff $w^{c}\left(\beta_{T}\right)$, representing the forecaster's continuation value from the reputation he has accumulated. We assume that $w^{c}(\cdot)$ satisfies the same requirements that we impose on $w(\cdot)$. Payoffs are interpreted as coming from some external source rather than the observer directly. A highly regarded analyst can bargain higher wage from employers in the labor market, while all of the interested public acts as the observer in forming analyst's reputation.

Communication. In any period $t \in \mathcal{T}$ the forecaster can send a report $m \in\{G, B\}$ to the observer, indicating his prediction about state $\omega$. The report is not verifiable, i.e., the forecaster's private information is not ever observable and/or contractible. Additionally, we assume that the forecaster can send at most one report throughout the game 13

\subsection{Timing}

At time $t=0$, the state of the world $\omega$ and the forecaster's type $\gamma$ are realized; forecaster's private signal realization $\eta^{*}$ and signal arrival time $t^{*}$ are drawn from respective distributions. After that, in every period $t \in\{1, \ldots, T-1\}$ the stage game proceeds as follows:

1. If $t=t^{*}$ and the forecaster is competent, he observes the realization of $\eta^{*}$;

2. The forecaster updates his belief about the state conditional on observed $\eta^{*}$ (if any) and decides whether to send a report $m \in\{G, B\}$ to the observer;

3. The observer updates her beliefs about the state $p$ and about the forecaster's competence $b$ conditional on the forecaster's report or lack of thereof;

4. The forecaster receives payoff $w\left(\beta_{t}\right)$;

In period $T$ steps 1 and 2 take place as above, but instead of steps 3 and 4 the following happens:

3. State $\omega$ is publicly revealed;

\footnotetext{
${ }^{12}$ In the discussion surrounding the model, we assume that she is interested in information about state. To fix ideas, one may think that the observer chooses a binary action from $\{G, B\}$ at time $T$ and receives a fixed reward if and only if her action matches the state - but we do not model this decision explicitly.

${ }^{13}$ This constraint should not be seen as restrictive since the forecaster receives at most one private signal by time $T$.
} 
4. All players update their beliefs accordingly;

5. The forecaster receives a terminal lump-sum payoff $w^{c}\left(\beta_{T}\right)$.

\subsection{Histories and State Variables}

A message history is $\mu_{t}=(m, s)$ if report $m$ has been made in period $s \leqslant t$ and $\mu_{t}=\varnothing$ otherwise. A public history $h_{t}^{p}$ is a tuple consisting of the variables that are publicly observable at the beginning of period $t: h_{t}^{p}=\left(t, \mu_{t-1}\right)$. The forecaster possesses private information about his type and his private signal in addition to whatever is publicly known. We define a type- $\gamma$ forecaster's private history as $h_{t}^{\gamma}=\left(h_{t}^{p}, \eta_{t}^{\gamma}, t^{\gamma}\right)$, where $\eta_{t}^{\gamma}$ describes forecaster's private information:

- $\eta_{t}^{\gamma}=\varnothing$ if no signal was observed in period $t$ or before,

- $\eta_{t}^{\gamma}=G$ if signal $\eta^{*}=G$ was observed in period $t$ or before,

- $\eta_{t}^{\gamma}=B$ if signal $\eta^{*}=B$ was observed in period $t$ or before.

Variable $t^{\gamma}$ indicates the arrival time of this information, with $t^{\gamma}=0$ meaning no information has yet arrived. For quacks we have that $t^{Q}=0$ and $\eta^{Q}=\varnothing$. For experts these variables can be expanded as $t^{E}=t^{*} \cdot \mathbb{I}\left(t \geqslant t^{*}\right)$, and $\eta^{E}=\eta^{*}$ if $t \geqslant t^{*}$, and $\eta^{E}=\varnothing$ otherwise. Notably, values $\left(\eta_{t}^{\gamma}, t^{\gamma}\right)$ are only nontrivial for the expert, thus the quack's private histories are equivalent to public histories, and hereinafter we will treat them as such. We also let $-\eta$ and $-m$ denote the "opposites" of $\eta$ and $m$ respectively: e.g., if $\eta=G$ then $-\eta=B$.

\subsection{The forecaster's Problem}

At every history the forecaster decides whether to send a report and, if yes, which report to send. The forecaster's pure strategy is thus a mapping from private histories $h_{t}^{\gamma}$ to the set of feasible messages (which equals $\{\varnothing, G, B\}$ if no report has yet been made and $\{\varnothing\}$ otherwise, since we restrict forecaster to sending at most one message throughout the game). The forecaster's mixed strategy is, as usual, a probability distribution over pure strategies. To simplify the analysis, the following restriction is imposed on strategies:

Assumption (Amnesia). At any pair of histories $\bar{h}_{t}^{E}, \overline{\bar{h}}_{t}^{E}$ which differ only in signal arrival times $\bar{t}^{*}<\bar{t}^{*} \leqslant t$, the strategy of the expert must be the same.

This assumption requires that after the private signal is observed, the expert's reporting strategy does not depend on its arrival time $t^{*}$. One may think of this as the expert not remembering when he received the information (but the information itself is never forgotten). This restriction bans strategies like "send a report two periods after receiving a signal". This, however, should not be considered a loss of generality, as the timing of signal arrival is neither observable by anyone except the expert, nor payoff-relevant for any player, so can be seen as nothing more than the expert's private randomization device.

Amnesia together with the fact that only histories with $\mu_{t-1}=\varnothing$ involve non-trivial choice of message allow us to define strategies on the smaller space of tuples $(t, \eta)$ rather than on all private histories $h_{t}^{\gamma}=\left(t, \mu_{t-1}, \eta_{t}^{\gamma}, t^{\gamma}\right)$. Therefore, we introduce the forecaster's behavioral strategy 
as $r_{\eta}^{\gamma}(m, t)$, which denotes the probability of forecaster $\gamma$ making report $m$ at time $t$ conditional on having private information $\eta=\eta_{t}^{\gamma}$ and having not made any report prior to $t$.14 Finally, denote $r^{\gamma}(m, t):=\mathbb{E}_{\eta} r_{\eta}^{\gamma}(m, t)$. It represents the hazard rate of report $(m, t)$ as perceived by the observer who does not possess the forecaster's private information $\eta$ (but these objects are still conditional on the forecaster's type).

The forecaster's optimization problem is hence as follows: at every private history $h_{t}^{\gamma}$ such that no report has yet been made $\left(\mu_{t-1}=\varnothing\right)$, the forecaster of type $\gamma \in\{E, Q\}$ chooses a continuation reporting strategy $\left\{r_{\eta}^{\gamma}(m, s)\right\}_{s \geqslant t}$ as a solution to the following problem:

$$
V_{t, \eta}^{\gamma}:=\max _{r_{\eta}^{\gamma}} \mathbb{E}\left[\sum_{s=t}^{T-1} w\left(\beta\left(h_{s}^{p}\right)\right)+w^{c}\left(\beta\left(h_{T}^{p}\right)\right) \mid t, \eta, \mu_{t-1}=\varnothing\right]
$$

subject to evolution of $\beta\left(h_{s}^{p}\right)$ described in the following subsection. The expectation is taken over all future histories. We also introduce a shorthand notation for the forecaster's continuation value from making report $m$ in period $\tau$ at history $h_{t}^{\gamma}$ :

$$
W_{t, \eta}^{\gamma}(m, \tau):=\mathbb{E}\left[\sum_{s=t}^{T-1} w\left(\beta\left(h_{s}^{p}\right)\right)+w^{c}\left(\beta\left(h_{T}^{p}\right)\right) \mid t, \eta, \mu_{\tau}=(m, \tau)\right] .
$$

With this notation we have that report $(m, t)$ is optimal at $t$ if and only if $V_{t, \eta}^{\gamma}=W_{t, \eta}^{\gamma}(m, t)$. Moreover, we use $W_{t, \eta}^{\gamma}(\varnothing)$ to denote the respective value from not making any report until the end of period $T$ (i.e., conditional on $\mu_{T}=\varnothing$ ). Finally, as the quack never receives the private signal, we suppress subscript $\eta$ when talking about $V_{t, \eta}^{Q}$ and $W_{t, \eta}^{Q}(m, \tau)$, and refer to these objects as $V_{t}^{Q}$ and $W_{t}^{Q}(m, \tau)$ respectively.

\subsection{Beliefs}

Two important characteristics of any public history $h_{t}^{p}$ are public beliefs about the type of the forecaster and about state of the world, $b\left(h_{t}^{p}\right)$ and $p\left(h_{t}^{p}\right)$. Recall that $h_{t}^{p}=\left(t, \mu_{t-1}\right)$. We will use this together with the structure of our model to introduce the following labels for beliefs:

$$
\begin{aligned}
b(m, t) & :=b(s,(m, t)) \\
b_{t} & :=b(t, \varnothing)
\end{aligned}
$$

$$
\begin{aligned}
p(m, t) & :=p(s,(m, t)) \\
p_{t} & :=p(t, \varnothing)
\end{aligned}
$$

for all $s \geqslant t$. In this notation, $b(m, t)$ is the belief about the forecaster's type held by the observer at any time $s \geqslant t$ conditional on report $m$ made at time $t$, and $b_{s}$ is the same belief held in the absence of any reports. The same applies for the observer's belief about state, and we will use the same notation for $\rho$ and $\beta$, where applicable. This notation is well defined because once a report has been made, both beliefs are frozen in place since no further information can be conveyed from the forecaster to the public.

Finally, we let $b^{\omega}(m, t)$ denote the belief about the forecaster's type given a terminal history $h_{T}^{p}=(T,(m, t))$ and given that the state was revealed to be $\omega$.

\footnotetext{
${ }^{14}$ This is a game of perfect recall, hence by Kuhn's theorem behavioral and mixed strategies are equivalent.
} 


\subsection{Equilibrium Definition}

We are looking for Weak Perfect Bayesian Equilibria of the game, which consist of a strategy profile $\left\{r_{\eta}^{\gamma}(m, t)\right\}$ and a belief profile $\left(b\left(h_{t}^{p}\right), p\left(h_{t}^{p}\right)\right)$ such that:

1. strategies $r_{\eta}^{\gamma}$ solve (3) given the observer's updating rule for $b\left(h_{t}^{p}\right)$,

2. all players update their beliefs via Bayes' rule on path.

We further adopt three following refinements (in addition to restriction to amnesiac strategies):

(OP) Off-path Pessimism: off the equilibrium path the beliefs are $p=p_{0}$ and $b=0$, with the exception that extreme belief $b=1$ is not updated;

(ML) Message Labeling: $r_{G}^{E}(G, t) \cdot r_{B}^{E}(B, t) \geqslant r_{G}^{E}(B, t) \cdot r_{B}^{E}(G, t)$ for any $t$;

(SY) Symmetry: $r_{G}^{E}(G, t)=r_{B}^{E}(B, t)$ and $r_{G}^{E}(B, t)=r_{B}^{E}(G, t)$ for all $t$.

Off-path pessimism (OP) only makes it easier to sustain any given strategy profile as equilibrium because it makes deviations extremely unappealing for the forecaster. In particular, if there is some PBE with some off-equilibrium path beliefs, then the same profile of strategies and on-path beliefs would still constitute a PBE when paired with off-path beliefs prescribed by (OP). The exception in (OP) comes into play only if $\pi=1$ (i.e., expert's information predicts the state perfectly) and only at histories at which the report was supposedly made by an informed expert for sure, but turned out to be incorrect. The exception says that the forecaster is then still believed to be competent. This behavior of beliefs can be explained as the limiting case of the model as $\pi \rightarrow 15$

Message labeling (ML) requires that report $m$ is more indicative of state $\omega=m$ than the other report. This assumption is without loss, since at any history $h_{t}^{p}$ we can assign message labels $G$ and $B$ to the two messages in such a way that (ML) is satisfied.

The only requirement that imposes any actual restrictions is symmetry (SY). It requires that the expert treats states and messages equally - if he has evidence of state $G$, he sends report $G$ with the same probability that he would have sent report $B$ if he had evidence of state $B$. This assumption is made for tractability, so that the observer's belief about state stays at a constant level $p=p_{0}$ as long as no report is made ${ }^{16}$ We have no reasons to believe that the predictions of our model would not hold in asymmetric equilibria.

\section{Equilibrium Analysis}

This section strives to characterize the set of all Weak PBE of the game. The main question that is answered in this section is as follows: assuming that in some equilibrium reports are only made at some set of periods $S \subseteq \mathcal{T}$, how do the forecaster's strategies look and how does the informativeness of the reports change across different periods? It turns out that all equilibria have quite a lot of common structure. Proofs of all statements presented in this chapter can be found in the Appendix.

\footnotetext{
${ }^{15}$ Section 6.3 discusses alternatives to $(\mathrm{OP})$ in case of perfect signals.

${ }^{16}$ Silence is informative about the state only if the expert conceals his private signals, and does so differently conditional on different information. Assumption (SY) explicitly prohibits the latter part of this.
} 


\subsection{Belief Updating}

This section specifies how exactly observer's beliefs $b$ and $p$ evolve given the forecasters' strategy profile $\left\{r_{\eta}^{\gamma}(m, t)\right\}$.

Conditional on the forecaster not making a report, the observer's beliefs are updated as follows:

$$
\beta_{t}=\beta_{t-1} \cdot \frac{1-r^{E}(G, t)-r^{E}(B, t)}{1-r^{Q}(G, t)-r^{Q}(B, t)}
$$

Conditional on no report by the end of period $T$ and realized state $\omega$, the observer's terminal belief is

$$
\beta^{\omega}(\varnothing)=\beta_{0} \cdot \prod_{t=1}^{T}\left(\frac{1-\sum_{m \in\{G, B\}} \mathbb{E}\left[r_{\eta}^{E}(m, t) \mid \omega\right]}{1-\sum_{m \in\{G, B\}} r^{Q}(m, t)}\right),
$$

which in a symmetric equilibrium reduces to $\beta^{\omega}(\varnothing)=\beta_{T}$.

Similarly, employing the Bayes' rule we can derive the observer's belief $\beta(m, t)$ following forecaster's report $(m, t)$, and the observer's terminal belief $\beta^{\omega}(m, t)$ given forecaster's report $(m, t)$ and the realized state $\omega$ :

$$
\begin{aligned}
\beta(m, t) & =\beta_{t-1} \cdot \frac{r^{E}(m, t)}{r^{Q}(m, t)}, \\
\beta^{\omega}(m, t) & =\beta_{t-1} \cdot \frac{\mathbb{E}_{\eta}\left[r_{\eta}^{E}(m, t) \mid \omega\right]}{r^{Q}(m, t)} .
\end{aligned}
$$

Finally, another relevant belief is the observer's belief about the current state, $p_{t}$. As mentioned before, symmetry implies that in the absence of the report this belief is frozen at its initial level, $p_{t}=p_{0}$. Following report $(m, t)$ the belief is updated as:

$$
\rho(m, t)=\rho_{t-1} \cdot \frac{\left(1-b_{t-1}\right) \cdot r^{Q}(m, t)+b_{t-1} \cdot \mathbb{E}_{\eta}\left[r_{\eta}^{E}(m, t) \mid \omega=G\right]}{\left(1-b_{t-1}\right) \cdot r^{Q}(m, t)+b_{t-1} \cdot \mathbb{E}_{\eta}\left[r_{\eta}^{E}(m, t) \mid \omega=B\right]}=\rho_{t-1} \cdot \frac{1+\beta^{G}(m, t)}{1+\beta^{B}(m, t)}
$$

\subsection{Supports of the Reporting Times}

Given $\gamma \in\{E, Q\}$ and $m \in\{G, B\}$, define support $\mathcal{S}:=\left\{t_{1}, t_{2}, \ldots, t_{|\mathcal{S}|}\right\} \subseteq \mathcal{T}$ as the set of times $t$ at which any report is made: 17

$$
\mathcal{S}:=\left\{t \in \mathcal{T} \mid r^{\gamma}(m, t)>0 \text { for some } \gamma, m\right\}
$$

Proposition 1. In any equilibrium, any report $(m, t)$ for $m \in\{G, B\}$ is made with positive probability by a quack if and only if it is ever made by an expert: $r^{E}(m, t)>0$ if and only if $r^{Q}(m, t)>0$.

The reasoning behind this proposition is as follows. Suppose that there exists $(m, t)$ such that $r^{Q}(m, t)>0$ but $r^{E}(m, t)=0$, i.e., report $m$ at $t$ is only ever made by a quack. Then after report $(m, t)$ the observer infers that the forecaster is surely incompetent. This renders report $(m, t)$ to be a dominated reporting strategy for the forecaster - strictly so if we recall that the belief about the

\footnotetext{
${ }^{17}$ More generally, the support $\mathcal{S}$ is a subset of public histories $h_{t}^{p}$ for which $r_{\eta}^{\gamma}(m, t)>0$ for some $\gamma, \eta, m$. Since a public history in our model consists of current time $t$ and a messaging history $\mu_{t}$, and reports can only be made at histories with $\mu_{t}=\varnothing$, it is without loss to define the support as a set of times.
} 
forecaster's type is a martingale. Therefore, there must exist another continuation strategy at time $t$ - i.e., at the public history $h_{t}^{p}=(t, \varnothing)$ - that results in strictly positive reputation for at least one period. No forecaster is willing to play strictly dominated strategies, hence this cannot happen in equilibrium. A similar logic is in play in the opposite case - if $r^{E}(m, t)>0$ and $r^{Q}(m, t)=0$ for some $(m, t)$ - except then reporting $(m, t)$ is a strictly dominant strategy for any type of the forecaster since it yields the maximal possible reputation starting from $t$ for the rest of the game. Reporting $(m, t)$ is then strictly preferred by the quack to any other alternative, which again gives a contradiction 18

\subsection{Informative Reports and Babbling}

If report $(m, t)$ is made in equilibrium, this does not by itself mean that it contains any meaningful information about the state of the world or the type of the forecaster. Following Crawford and Sobel [1982], we refer to uninformative reports as babbling.

Definition. We say that report $(m, t)$ is babbling if

$$
\begin{aligned}
& b(m, t)=b_{t-1} \\
& p(m, t)=p_{t-1}
\end{aligned}
$$

Report $(m, t)$ is informative if it is not babbling.

Condition (9) implies that the report is uninformative about the forecaster's type, while (10) implies that it contains no information about the state.

It turns out that due to restriction that a forecaster can send at most one report, babbling reports in any equilibrium are organized in a specific structure. This is illustrated by the next proposition.

Proposition 2. Every equilibrium contains a Godwin point $\bar{t}:=\min \left\{t \in \mathcal{T} \mid V_{t, \varnothing}^{E}=V_{t}^{Q}\right\}$ such that:

1. All on-path reports $(m, t)$ with $t>\bar{t}$ are babbling.

2. No on-path reports $(m, t)$ with $t \leqslant \bar{t}$ are babbling. Moreover:

- at every $t<\bar{t}$ the expert does not make a report unless he has received the corresponding signal, i.e., $r_{\varnothing}^{E}(m, t)=0$ and $r_{\eta}^{E}(m, t)=0$ whenever $\eta \neq m$;

- at $t=\bar{t}$ the informed expert always reports his signal, i.e., $r_{\eta}^{E}(\eta, \bar{t})=1$.

"Godwin's law" states that as a discussion on the Internet continues for long enough, the probability of a comparison involving Nazis or Hitler approaches 119 At that point the informative part of the discussion is usually considered finished, and what follows is just babbling. Along similar lines, Proposition 2 says that in our model all equilibria feature at most two phases: early reports

\footnotetext{
${ }^{18}$ One may easily show using the same kind of argument that $r^{E}(G, t)+r^{E}(B, t)<1$ if and only if $r^{Q}(G, t)+$ $r^{Q}(B, t)<1$. I.e., a quack stays silent up until time $t$ with positive probability if and only if so does expert. See the proof of Proposition 1 for details.

${ }^{19}$ See "Meme, Counter-Meme" (Wired).
} 
are informative, while the late ones do not contain any relevant information about the state or the type of the forecaster.

To understand Proposition 2 it is enough to note that by Proposition $1, t \in \mathcal{S}$ only if an expert is willing to report at $t$. His comparative advantage relative to quack is his ability to acquire private signals. Therefore, the expert is only willing to participate in babbling if he has no option to exploit his [current or possibly future] information by sending an informative report - i.e., if the Godwin point $\bar{t}$ has passed and the discourse has descended into babbling. Conversely, whenever an option to make an informative report now or in the future is present (i.e., $t<\bar{t}$ ), the expert is not willing to report contrary to his private information or make an unfounded report. The only kind of information distortion that he is willing to partake in is delaying information revelation but even in this case delaying beyond the Godwin point $\bar{t}$ cannot be worth it.

It is worth noting that $\bar{t}$ does not have to be in the interior of the support, so one of the phases may be absent. In particular, if $\bar{t}<t_{1}$ then all reports are babbling, while if $\bar{t}=t_{|\mathcal{S}|}$ then no babbling takes place in equilibrium. We shall refer to the latter type of equilibria as informative.

Definition. An informative equilibrium is an equilibrium where all reports in the support are informative.

Note that in any informative equilibrium with support $\mathcal{S}$, it must be that $\bar{t}=t_{|\mathcal{S}|}$, since the definition directly implies $\bar{t} \geqslant t_{|\mathcal{S}|}$, and the condition $V_{t, \varnothing}^{E}=V_{t}^{Q}$ is satisfied for $t=t_{|\mathcal{S}|}$.

The next proposition shows that the babbling phase may be safely ignored altogether, and without loss of generality we may consider only informative equilibria.

Proposition 3 (Babbling Irrelevance). For any equilibrium with support $\mathcal{S}$ and Godwin point $\bar{t}$ there exists an informative equilibrium with the same Godwin point $\bar{t}$ and support $\tilde{\mathcal{S}}=\mathcal{S} \cap\{t \leqslant \bar{t}\}$ such that the two equilibria are:

1. payoff-equivalent for all players,

2. strategy-equivalent on $\tilde{\mathcal{S}}$.

Propositions 2 and 3 together imply that any equilibrium strategy profile with some Godwin point $\bar{t}$ can be obtained from a respective informative equilibrium with the same Godwin point by allowing for some babbling in $\{\bar{t}+1, \ldots, T\}$.

Finally, to simplify the statements of our results, we will also focus on reticent equilibria, as defined below.

Definition. We call an equilibrium reticent if $r_{\varnothing}^{E}(G, \bar{t})=r_{\varnothing}^{E}(G, \bar{t})=0$.

In informative reticent equilibria, we then have that for all $t \in \mathcal{S}: r_{\varnothing}^{E}(m, t)=0$ for all $m \in\{G, B\}$ and $r_{\eta}^{E}(m, t)=0$ for $\eta \neq m$. The expert in such equilibria only makes a prediction $m$ if he has received private signal $\eta=m$. The main remaining question is how the quack responds to such an expert's strategy. The following subsection answers this question in the context of informative reticent equilibria, and in Section 6.2 we show how these results extend to equilibria that are not reticent. 


\subsection{Main Results}

This section fixes an arbitrary support $\mathcal{S}=\left\{t_{1}, t_{2}, \ldots, t_{|\mathcal{S}|-1}, t_{|\mathcal{S}|}=\bar{t}\right\} \subseteq \mathcal{T}$ and explores properties of informative reticent equilibria on $\mathcal{S}$ (assuming they exist). Other kinds of equilibria are explored in Section 6. For simplicity we also assume throughout the remainder of Section 5 that the expert's signals are absolutely precise $(\pi=1)$; this assumption is relaxed in Section 6.4.

To start with, it is useful to understand how equilibria look conditional on the support. Propositions 2 and 3 imply that the the expert only reports when he has already received a private signal, except maybe at the last point of the support. Assumption (SY) then implies that if $r^{E}(G, t)>0$ then $r^{E}(B, t)>0$ and vice versa. Proposition 1 together with the above leads to the fact that in any informative equilibrium for any $t \in \mathcal{S}$ : (1) both reports $m=G$ and $m=B$ are made at $t$ in equilibrium, and (2) both types of forecasters make any given report $m \in\{G, B\}$ at $t$ in equilibrium. Alternatively, one may say that $\mathcal{S}=\left\{t \mid r_{\eta}^{E}(\eta, t)>0\right\}$ for any $\eta$, i.e., in any informative equilibrium the support is a set of times at which the expert discloses some of the information he possesses.

To talk about the informativeness of different predictions about the state of the world, we introduce the following measure:

$$
i(m, t):=\ln (\rho(m, t))-\ln \left(\rho_{t-1}\right)=\ln \left(1+\beta^{G}(m, t)\right)-\ln \left(1+\beta^{B}(m, t)\right){ }^{20}
$$

This measure shows how likely report $(m, t)$ is to be sent in state $G$ as opposed to state $B$. Positive values reinforce the observer's belief in state $\omega=G$ after hearing this report, while negative values do the same for state $\omega=B$. Higher absolute values of $i(m, t)$ mean that more information is transmitted by message $(m, t)$ to the observer, meaning that belief $\rho(m, t)$ moves further away from $\rho_{t-1}$.

Presented next is the central result of our paper, which describes the informational content of reports and the informativeness dynamics. All monotonicity statements in this Theorem are understood in the sense of weak monotonicity.

Theorem 1. Suppose that $|\mathcal{S}| \geqslant 2$ and an informative reticent equilibrium on $\mathcal{S}$ exists. Then in any such equilibrium the following are true for both $m \in\{G, B\}$ :

1. later reports are less informative about the state: $|i(m, t)|$ is a decreasing function of $t$ on $\mathcal{S}$;

2. the reputation of a silent forecaster improves over time: $b_{t}$ is increasing in $t$ on $\mathcal{S}$ and constant on $\mathcal{T} \backslash \mathcal{S}$;

3. making any report decreases reputation as compared to no report: $b(m, t) \leqslant b_{t}$ for any $t \in \mathcal{S}$.

Theorem 1 starts by stating that in any reticent informative equilibrium with $|\mathcal{S}| \geqslant 2$ reports should become [weakly] noisier over time. This is required to provide incentives for the expert to disclose the information he possesses. To elaborate, Proposition 1 implies that a quack must be indifferent between all reports $(m, t)$ made in equilibrium. At the same time, the only difference

\footnotetext{
${ }^{20}$ Note that since Bayes' rule is linear in log-likelihoods, $|i(m, t)|$ shows exactly the "strength" of the signal contained in $(m, t)$ in terms of it's effect on the posterior $p(m, t)$ relative to the prior $p_{t-1}$. That said, our results are not specific to the particular functional form of $i(m, t)$ and are compatible with any other measure of distance between $\rho(m, t)$ and $\rho_{t-1}$ which is increasing in $\left|\rho_{t-1}-\rho(m, t)\right|$ for any fixed $\rho(m, t)$.
} 
between the expert's and the quack's payoffs comes from their respective probabilities of guessing the state correctly with their report. Therefore, conditional on the quack's indifference, the expert with information $\eta \in\{G, B\}$ in period $t$ effectively maximizes the net premium for guessing the state correctly, as given by

$$
\Delta w_{\eta}(m, \tau):=w^{c}\left(\beta^{\eta}(m, \tau)\right)-w^{c}\left(\beta^{-\eta}(m, \tau)\right),
$$

over all reports $(m, \tau)$ with $\tau \geqslant t$. From (ML) and (SY) we know that $\Delta w_{\eta}(m, \tau)$ is weakly positive for $m=\eta$ and is weakly negative for $m=-\eta$, hence it is enough to consider $m=\eta$. Moreover, Propositions 1 and 2 together imply that in informative equilibria, $t \in \mathcal{S}$ if and only if an informed expert reports at $t$. This means that for $t \in \mathcal{S}$ we have

$$
(\eta, t)=\max _{m, \tau \in \mathcal{S}, \tau \geqslant t} \Delta w_{\eta}(m, \tau)
$$

or, simply speaking, $\Delta w_{\eta}(m, t)$ must be a weakly decreasing function of $t$ on $\mathcal{S}$ for $m=\eta$. Note that in case $\pi=1$, Proposition 2 implies that $\beta^{-\eta}(\eta, t)=0$ for all $t \in \mathcal{S} \backslash\{\bar{t}\}$, and therefore $\Delta w_{\eta}^{c}(\eta, t)=w\left(\beta^{\eta}(\eta, t)\right)$. Finally, as $w(\cdot)$ is strictly increasing, its monotonicity is equivalent to monotonicity of $\beta^{\eta}(\eta, t)$, which in the end directly translates into that of $|i(\eta, t)|$.

The second and the third statements can be shown using the monotonicity of $\beta^{m}(m, t)$ derived above, but the main intuition behind them comes from the quack's indifference between all reports made in equilibrium. Take some $t_{k} \in \mathcal{S}$ and suppose that $b\left(m, t_{k}\right)<b_{t_{k}}$ for both $m \in\{G, B\}$. Then it should be that $b\left(m, t_{k+1}\right)<b\left(m, t_{k}\right)$ for both $m$, since otherwise any report $\left(m, t_{k}\right)$ dominates any report $\left(m, t_{k+1}\right)$ - the former grants higher payoff at $t_{k}$, higher payoff between $t_{k+1}$ and $T$, and higher continuation payoff after $T$. By the martingale property of beliefs, $b\left(m, t_{k+1}\right)$ and $b_{t_{k+1}}$ should average out to $b_{t_{k}}$, so in the end we have that

$$
b\left(m, t_{k+1}\right)<b\left(m, t_{k}\right)<b_{t_{k}}<b_{t_{k+1}}
$$

whenever $b\left(m, t_{k}\right)<b_{t_{k}}$. The same argument extends to all $t \in \mathcal{S}$, granting the second and third statements of Theorem 1. This argument does not preclude monotonicity from going the other way if we start from the inequality $b\left(m, t_{k}\right)>b_{t_{k}}$ - but this case would generate a sequence $b^{m}(m, t)$ that is increasing in $t \in \mathcal{S}$, which is incompatible with the expert's preferences discussed previously. Finally, the argument above implies that penalties for reporting increase over time: if $t_{k}, t_{k+1}, t_{k+2} \in \mathcal{S}$ then

$$
b_{t_{k+1}}-b\left(m, t_{k+2}\right)>b_{t_{k}}-b\left(m, t_{k+1}\right) .
$$

This is exemplified in Figure 3, where the red solid line shows the reputation path of a forecaster who makes a report at $t=3$, and the blue dashed line shows that for $t=4$.

Finally, it is worth noting that even the third statement, which is inherently static, requires $|\mathcal{S}| \geqslant 2$. If $|\mathcal{S}|=1$ (so $\mathcal{S}=\{\bar{t}\}$ ) then it is no longer true: one may construct an equilibrium with $b(m, \bar{t})>b_{\bar{t}}$ for both $m \in\{G, B\}$. In such equilibrium either report is more likely to be made by an expert than a quack. "Static" equilibria (those with $|\mathcal{S}|=1$ ) are in this sense potentially more informative than "dynamic" equilibria, and allowing for reports to be made at more than one point in time may actually be harmful to the informativeness of these reports. 


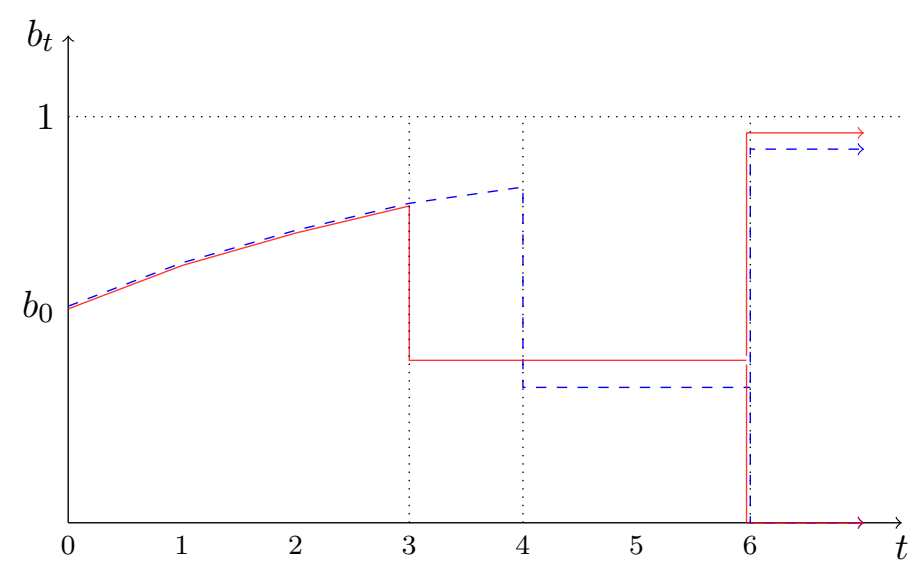

Figure 3: Report penalties increase over time.

\subsection{Existence of Informative Equilibria}

So far we have discussed properties of equilibria without proving that any equilibria actually exist, but existence of informative equilibria is not a trivial concern ${ }^{21}$ The following Proposition outlines some necessary and sufficient conditions for existence of informative equilibria, which allow to understand some driving forces behind their existence and non-existence.

Proposition 4. Suppose $w(\cdot)$ and $w^{c}(\cdot)$ are continuous function. Then

1. For any $\bar{t}$ there exists an informative equilibrium with $\mathcal{S}=\{\bar{t}\}$;

2. If $w(\beta)$ and $w^{c}(\beta)$ are convex and $p_{0}=\frac{1}{2}$ then an reticent informative equilibrium with arbitrary $\mathcal{S}$ exists;

3. If $w(\beta)=\beta^{\alpha}$ and $w^{c}(\beta)=\theta \beta^{\alpha}$ with $\theta>0$ and $\alpha<1$, then no informative equilibrium with $|\mathcal{S}| \geqslant 3$ exists.

Part 1 of Proposition 4 states that at least some informative equilibria always exist. In particular, there always exist equilibria with singleton support, whatever the single period in the support is. At this period experts reveal all private information they have accumulated by then, and any forecaster without private information is also free to make a report in the hopes of guessing the state correctly.

However, the main focus of this paper is on the dynamics of announcements, so we are particularly interested in equilibria with $|\mathcal{S}|>1$. Part 2 of Proposition 4 gives a sufficient condition for their existence, which is convexity of the payoff function $w(\cdot)$ and symmetry of the two states, $p_{0}=\frac{1}{2}$. By the continuity of payoffs, the condition on $p_{0}$ can be relaxed to some extent. All else equal, for any convex $w^{c}(\cdot)$ there exists $\varepsilon>0$ such that an informative equilibrium for arbitrary support $\mathcal{S}$ exists whenever $p_{0} \in\left(\frac{1}{2}-\varepsilon, \frac{1}{2}+\varepsilon\right)$.

Necessary conditions, on the other hand, are not easily obtainable in our model. The reason lies in the fact that the payoff functions $w(\cdot)$ and $w^{c}(\cdot)$ are only invoked for a finite number of arguments $\beta$ in any given equilibrium. In particular, given some payoff function $w(\cdot)$ and some equilibrium of the game, we can change values that $w(\cdot)$ and/or $w^{c}(\cdot)$ take almost everywhere without affecting the equilibrium. This makes necessary conditions difficult to formulate without restricting payoff

\footnotetext{
${ }^{21}$ Babbling equilibria, on the other hand, always trivially exist.
} 
functions to a specific class, which is what the last part of Proposition 4 does. It states that for at least some class of concave payoff functions the existence of equilibria with large supports $(|\mathcal{S}| \geqslant 3)$ completely breaks down 22 Parts 1 and 3 together illustrate that the main hurdles to existence are tied to intertemporal choice: if the forecaster has no choice of when to make a report then existence is certain, while allowing predictions to be made at multiple points in time may in some settings lead to complete breakdown of communication.

The reason for non-existence is connected to the expert's dynamic incentive compatibility constraints. This is because $t \in \mathcal{S}$ if and only if the informed expert makes a report at $t$, so he should be willing to do so instead of delaying his report until a later date. This leads to phenomena described in parts 2 and 3 of Theorem 1 1 . In particular, any report has to reduce the forecaster's reputation, so the only reason to make the report for the quack is a gamble for the terminal reputation: he should be willing enough to make a guess, understanding that it may be incorrect. A certain degree of risk-loving on behalf of the forecaster is required for such a strategy profile to constitute an equilibrium. Conversely, if the quack is too risk-averse then strategy profiles with $|\mathcal{S}| \geqslant 3$ cannot satisfy the incentives of both forecaster types at the same time, ${ }^{23}$ The formal argument is somewhat more subtle and can be found in the Appendix.

The intuition above naturally leads to the question: do there exist equilibria, given enough risk-aversion on forecaster's behalf, in which the quack is too reluctant to make his report for fear of guessing it wrong? Such equilibria do not require sustaining quack's indifference between all reports, so they should seemingly exist under a wider range of parameters and functional forms. In the current setting the existence of such equilibria violates Proposition 1 and is therefore impossible. In Section 6.3 we show that after adopting an alternative assumption on off-path beliefs such equilibria can in fact exist, but only if $\pi=1$.

\subsection{Comparison of Equilibria}

In this section we study how the informativeness of the reports depends on the shape of equilibrium. Simply speaking, we are trying to answer the question of which equilibria are more informative.

We have two characteristics that describe how informative a given equilibrium is: its support $\mathcal{S}$ and two functions $i(m, t)$ for $m \in\{G, B\}{ }^{24}$ Their exact meaning, however, is worth clarifying. The informativeness measure $|i(m, t)|$ is effectively a signal-to-noise ratio: it shows how noisy a given message is, conditional on the event that this message is sent. The probability of the latter, however, is governed by $\mathcal{S}$, so $|i(m, t)|$ alone does not allow to conclude ex ante how much information will be conveyed at $t$. Sparser support $\mathcal{S}$ means that reports arrive more rarely in equilibrium and it may take longer for a given piece of information to be disclosed, but it does not necessarily imply that less information is transmitted (as long as $\bar{t}$ is unaffected). To elaborate, any piece of information that is observed by the expert at $t^{\prime} \notin \mathcal{S}$ is not lost to the void - its revelation is delayed until

\footnotetext{
${ }^{22}$ The jump from $|\mathcal{S}|=1$ to $|\mathcal{S}| \geqslant 3$ is tied to the special features of the Godwin point, which precludes us from making sharp statements about equilibria with $|\mathcal{S}|=2$. See also Section 6.2

${ }^{23}$ Remember that $w\left(\beta_{t}\right)$ is a function of $\beta_{t}=\frac{b_{t}}{1-b_{t}}$ which itself is a convex function of $b_{t}$. Therefore, even with $\alpha=1$ the forecaster is still risk-loving, so all talks of risk-loving and risk-aversion are in the relative sense (one may easily verify that coefficients of both absolute and relative risk-aversion are monotone in $\alpha$ ).

${ }^{24}$ This discussion implicitly focuses on the observer's welfare. forecaster's type is not of interest to the observer, hence $g(m, t)$ is not a variable of interest for us.
} 
$t^{\prime \prime}=\min \left\{t \in \mathcal{S} \mid t>t^{\prime}\right\}$ but it is reported eventually.

Proposition 5 below summarizes our knowledge of how different equilibria of the game compare to each other in terms of informativeness, given some fixed underlying fundamentals.

Proposition 5. Assume that two reticent informative equilibria exist with respective supports $\mathcal{S}=\left\{t_{1}, \ldots, t_{k}\right\}$ and $\tilde{\mathcal{S}}=\left\{t_{1}, \ldots, t_{k}, t_{k+1}, \ldots, t_{k+n}\right\}$, and informativeness measures $i(m, t)$ and $\tilde{i}(m, t)$. Then $|i(m, t)| \leqslant|\tilde{i}(m, t)|$ for $m \in\{G, B\}$ and all $t \in \tilde{\mathcal{S}}$.

The proposition says that expanding support to the right increases the informativeness of all reports as long as the expert makes no uninformed reports. In other words, this says that "extending the deadline" for reports - in the sense of switching to an equilibrium with larger $\mathcal{S}$ - is always good for the observer. It both allows more information to be transmitted by the informed expert (in case he observes his private information between $t_{k}$ and $t_{k+n}$ ) and decreases noise of all informative reports (weakly for all $t \leqslant t_{k}$ and strictly for all $t>t_{k}$ ). The intuition behind the latter phenomenon follows from Theorem 1. Simply speaking, the more reporting options are available to quack in a given equilibrium, the thinner he spreads over them. A more detailed argument follows.

Ceteris paribus, extending the support to the right (i.e., adding later dates) implies that the reputation $b_{t}$ of the silent forecaster should improve at the new dates. This makes an option of staying silent (or making a report at the last point) more attractive to the quack and does not affect his payoff from making a report. By Proposition 1, the quack should be indifferent between these options, so to restore this indifference after expanding the support we have to make reports more appealing to him - which is achieved by prescribing point-wise lower $r^{Q}(m, t)$ in equilibrium, thereby improving $b(m, t)$ and $b^{\omega}(m, t)$ and at the same time depressing $b_{t}$.

\section{Discussion and Extensions}

\subsection{Delay Equilibria}

Although Proposition 2 states that the expert only reports at $t<\bar{t}$ if he has already received a signal, it is still possible that he may delay his report, making it some time after he has received a signal (but no later than $\bar{t}$ ). If this happens, we call an equilibrium a delay equilibrium. Conversely, if the expert always discloses his information immediately then we call it a relay equilibrium.

Definition. We call an informative equilibrium:

1. a relay equilibrium if $r_{\eta}^{E}(\eta, t)=1$ for $\eta \in\{G, B\}$ and for all $t \in \mathcal{S}$;

2. a delay equilibrium otherwise.

Delay equilibria are very special in two respects. Firstly, unlike relay equilibria, they only exist under knife-edge conditions on parameters. In other words, a generic informative equilibrium is a relay equilibrium, in which the expert discloses his signals immediately. Secondly, delay equilibria necessarily possess more concrete properties than relay equilibria. In particular, Proposition 6 describes how the equilibrium properties described in Theorem 1 specialize in case of delay equilibria.

Proposition 6. Suppose that $|\mathcal{S}| \geqslant 2$ and an reticent delay equilibrium on $\mathcal{S}$ exists. Then in any such equilibrium the following are true for both $m \in\{G, B\}$ : 
1. the report informativeness $|i(m, t)|$ is constant for all $t \in \mathcal{S}$;

2. the silent forecaster's reputation is independent of time: $b_{t}$ is constant on $\mathcal{T}$;

3. the forecaster's reputation is not immediately affected by his report: $b(m, t)=b_{t}$ for any $t \in \mathcal{S}$.

Both observations above (that existence conditions and equilibrium properties of delay equilibria present a special case of those for relay equilibria) stem from a common source. In comparison to relay equilibria, delay equilibria impose an extra set of restrictions on players' payoffs: the informed expert must be indifferent between revealing his signal today and delaying his report until the next $t \in \mathcal{S}$. Given that this should be satisfied for both kinds of private signals together with quack's indifference, the set of compatible equilibrium belief profiles shrinks significantly which allows us to provide a significantly stronger version of Theorem 1 for delay equilibria.

\subsection{Informative Equilibria without Reticence}

When presenting the main result of the paper in Theorem 1 and in Proposition 6, we restricted the set of possible equilibria to reticent equilibria. The main result, however, holds without this assumption so long as we exclude $\bar{t}$ from parts 2 and 3 of the statement.

Proposition 7. Suppose that $|\mathcal{S}| \geqslant 3$ and an informative equilibrium on $\mathcal{S}$ exists. Then in any such equilibrium the following are true for both $m \in\{G, B\}$ :

1. later reports are less informative about the state: $|i(m, t)|$ is a decreasing function of $t$ on $\mathcal{S}$;

2. the reputation of a silent forecaster improves over time: $b_{t}$ is increasing in $t$ on $\mathcal{S} \backslash\{\bar{t}\}$ and constant on $\mathcal{T} \backslash \mathcal{S}$;

3. making any report decreases reputation as compared to no report: $b(m, t) \leqslant b_{t}$ for any $t \in \mathcal{S} \backslash\{\bar{t}\}$.

Proposition 7 differs from Theorem 1 and Proposition 6 in two respects: it requires $|\mathcal{S}| \geqslant 3$ and excludes $\bar{t}$ from statements 2 and 3. The common reason behind both of these changes is that the Godwin point $\bar{t}$ differs from other points in $\mathcal{S}$. Its distinctive feature is allowing $r_{\varnothing}^{E}(m, \bar{t})>0$ - that an uninformed expert makes a report, - while from Proposition 2 we know that $r_{\varnothing}^{E}(m, t)=0$ for all $t<\bar{t}$. This can generate situations in which statements 2 and 3 are no longer true at $\bar{t}$, i.e., some report $m$ may have $b(m, \bar{t})>b_{t}$, while silence would decrease $b_{t}$. This, however, does not affect the first part of the proposition: the reports made by the uninformed expert at $\bar{t}$ are uninformative, and thus only add more noise, amplifying the effect of decreasing informativeness as compared to reticent equilibria.

\subsection{Ideal Equilibria}

Informative equilibria with nontrivial supports need not exist with non-convex payoffs, as evidenced by Proposition 4. A question arises: are babbling and small-support equilibria the only possible outcomes when forecasters are too risk-averse? The answer is "not necessarily".

The key to answering this question is assumption $(\mathrm{OP})$. It requires that once a forecaster has gained perfect reputation it persists forever - even if a forecaster's prediction turned out to be wrong 
when it could not happen in equilibrium (which is the case if the expert is supposed to report in equilibrium only if he has the respective signal). This is a limiting case of the model as $\pi \rightarrow 1$, i.e., it can be supported by a perturbation of the model in which the expert's signal is incorrect with vanishing probability - and thus so are his predictions (see Section 6.4 for a more extensive discussion of this setting).

However, this is not the only possible perturbation of the model in case $\pi=1$. One may alternatively think of a version of the model with the infinitesimal number of "crazy" forecasters who are not strategic in their reports and just voice their opinions at random times. Since their number is infinitesimal, Bayes' rule still prescribes that $b(m, t)=1$ for any $(m, t)$ such that $r_{\eta}^{E}(m, t)>0=r^{Q}(m, t)=r_{\varnothing}^{E}(m, t)$ with $\eta=m$. However, since an informed expert is never wrong, if such prediction $(m, t)$ turns out incorrect, this would imply that it was actually made by one of the few crazy forecasters who may be competent or not. This could lead to any belief $b^{-m}(m, t) \in[0,1]$.

In this section we substitute (OP) by an alternative assumption (OP') which prescribes the worst possible off-path belief after an incorrect prediction supposedly made by an expert, same as any other off-path history:

(OP) off the equilibrium path the beliefs are $p=p_{0}$ and $b=0$, with the exception that the extreme belief $b=1$ is not updated;

(OP') off the equilibrium path the beliefs are $p=p_{0}$ and $b=0$.

The alternative assumption (OP') allows for the existence of ideal equilibria:

Definition. Ideal equilibria are characterized by $r^{Q}(m, t)=r_{\varnothing}^{E}(m, t)=0$ for all $(m, t), r_{\eta}^{E}(m, t)=0$ for $\eta \neq m$, and $r_{\eta}^{E}(m, t)>0$ for some $(m, t)$ with $m=\eta$.

Simply speaking, in ideal equilibria the only reports that are ever made are those by informed experts; quacks never voice their opinion. This type of equilibrium is enforced by the worst possible terminal reputation if the forecaster's report turned out incorrect. For this threat to enforce such an equilibrium, the quacks should be afraid of bad reputation more than they should love good reputation in the short term. In other words, the payoff from reputation $w(\cdot)$ must be relatively concave. While we cannot state the necessity of concavity (see Section 5.5 for discussion of necessary conditions), we can show the converse: if $w(\cdot)$ or $w^{c}(\cdot)$ is convex then ideal equilibria do not exist.

Proposition 8. Under $\left(O P^{\prime}\right)$, if $w(\cdot)$ or $w^{c}(\cdot)$ is convex then no ideal equilibria exist.

This is the exact opposite of part 2 of Proposition 4 meaning that ideal equilibria are, informally speaking, complementary to informative equilibria in the sense of existence. On the formal side, the proof of Proposition 8 contains the necessary and sufficient condition for existence of an ideal equilibrium with support $\mathcal{S}$, but this condition is not particularly insightful, and for that reason we do not state it here.

\subsection{Imperfect Private Signals}

In this section we relax the assumption that the expert's signals are perfectly informative about the state and explore the case $\pi<1$. Note that there is nothing in the intuition behind Theorem 
1 implying that $\pi=1$ is a necessary condition. As long as $\pi>\frac{1}{2}$, the expert's signal is somewhat informative about the state, so his informed report about the state is more likely to be supported by the ex post evidence than the quack's random guess. Therefore, the results should continue to hold.

The proofs of Propositions 1, 2, and 3 continue to hold in case $\pi<1$ with no further modifications. Proposition 9 below shows that the remaining results continue to hold as well if $w^{c}(\cdot)$ is either convex, or at least not too globally concave, and the private signal is sufficiently precise 25

Proposition 9. Theorem 1, Propositions 4- 7 are true for $\pi<1$ if either of the following holds:

1. $w^{c}(\cdot)$ is convex;

2. $w^{c}(\cdot)$ is continuously differentiable and there exist $0<\underline{d} \leqslant \bar{d}<+\infty$ such that $\frac{d w^{c}(\beta)}{d \beta} \in[\underline{d}, \bar{d}]$ and $\pi>\frac{\bar{d}}{\underline{d}+\bar{d}}$.

When describing the intuition behind Theorem 1, we have mentioned that in order to provide incentives for the informed expert to reveal his private information immediately instead of waiting for a later date, the premium $\Delta w_{\eta}(m, t)$ for guessing the state correctly should be a decreasing function of $t$ on $\mathcal{S}$. An important part of the proof of Theorem 1 consists of showing that decreasing $\Delta w_{\eta}(m, t)$ is equivalent to decreasing $b^{m}(m, t)$. The three statements of Theorem 1 then follow almost directly from the latter statement (using the Bayes' rule and the martingale property of beliefs).

The equivalence relation above is simple when $\pi=1$, since then $b^{-m}(m, t)=0$, and $w^{c}(\cdot)$ is a strictly increasing function. Proposition 9 provides two alternative conditions under which the equivalence holds in case $\pi<1$. If $w^{c}(\cdot)$ is convex it holds because $b^{m}(m, t)$ and $b^{-m}(m, t)$ are scalar multiples of each other ${ }^{26}$ The second condition relaxes convexity to just bounded derivative of $w^{c}(\cdot)$ but the idea is the same: if $\frac{d w^{c}(\beta)}{d \beta}$ is bounded so that $w^{c}(\cdot)$ is not too concave globally, and the signal is precise enough, we can establish the connection between $\Delta w_{\eta}(m, t)$ and $b^{m}(m, t)$.

It is also worth noting that ideal equilibria outlined in Section 6.3 can no longer exist if $\pi \in\left(\frac{1}{2}, 1\right)$. This is because the forecaster who is believed competent with probability one can no longer be punished after his prediction was revealed to be wrong - he can credibly claim that the mistake was made because of an incorrect private signal, rather than due to low competence.

\subsection{Commitment}

Suppose now that the forecaster can commit to a reporting strategy at $t=0$ after learning his type but before receiving any private information. The forecaster's strategy is not publicly observable. This modification relates our problem to the literature on Bayesian Persuasion and information design, since the forecaster now designs the disclosure strategy subject to the constraints on the information available to him 27

\footnotetext{
${ }^{25}$ The exception is Proposition 8, since assumption (OP') is equivalent to (OP) when $\pi<1$.

${ }^{26}$ This follows from the observer's belief $p_{t}$ regarding state being constant in the absence of reports and the rate of arrival of expert's private signal being the same in both states. Due to these assumptions, ratio of $b^{m}(m, t)$ to $b^{-m}(m, t)$ equals the relative probability of expert having correct versus incorrect information about the state.

${ }^{27}$ The seminal contribution is Kamenica and Gentzkow 2011; see Bergemann and Morris 2019 for a recent survey.
} 
The literature on Bayesian Persuasion has demonstrated that commitment power often allows the sender to strictly improve his payoff whenever the optimal communication mechanism is informative 28 In contrast, it is easy to see that in our setting all forecaster's strategy profiles that were optimal in the absence of commitment remain optimal even if he has commitment power. In particular, the quack is indifferent between all reports sent in equilibrium and not reporting, if allowed in equilibrium. This means that his payoff from any action played in equilibrium is the same, and (OP) implies that playing off-path actions is no better. Therefore, conditional on the expert's strategy, the quack cannot improve by committing to a different strategy. On the other hand, the expert's strategy is also optimal given the quack's indifference: given information $\eta$, it is optimal for him to send the report $(m, t)$ that maximizes $\Delta w_{\eta}(m, t)$, and given no information it is optimal to wait for information (until at least $\bar{t}$ ). Therefore, the forecaster's commitment power does not affect the equilibria identified above.

\section{Conclusion}

The paper presents a model of dynamic cheap talk in the presence of career concerns. We discover that the competition between competent (experts) and incompetent (quacks) forecasters imposes plenty of structure on equilibrium outcomes. In particular, we show that to incentivize the experts - whose reports drive the whole market, - to make early predictions, it must be that early reports are perceived more favorably by the public than later reports. Perhaps more surprisingly, we discover that the presence of quacks in the market together with the monotonicity above generates an automatic penalty for any report: a forecaster who makes a prediction will see his reputation plummeting, and he will only be redeemed if his prediction will turn out to be correct. This does not discourage quacks from speaking up, but disciplines their incentives. Moreover, this reputation dynamics implies that for non-trivial equilibria to exist, forecasters' payoffs must be sufficiently convex in reputation, which is the case if, e.g., the premium for being the top forecaster in the field is very large.

These predictions are novel in the literature, and are driven by us explicitly modeling the dynamic payoff structure of the forecasters. Our model accounts for both flow payoffs while the public is still uncertain about the correctness of the forecaster's prediction, and terminal payoffs realized after the true state is revealed.

The model can be extended in multiple directions, e.g., to account for competition among forecasters, or for arrival of public signal in the background. Richer private news processes for forecasters can also add another strategic layer to the timing decision of the forecaster's prediction. All of these are prospective avenues for future research.

\section{References}

V. V. Acharya, P. DeMarzo, and I. Kremer. Endogenous information flows and the clustering of announcements. American Economic Review, 101(7):2955-2979, December 2011.

C. Aghamolla and B.-J. An. Voluntary disclosure with evolving news. working paper, 2015.

\footnotetext{
${ }^{28}$ See Lipnowski, Ravid, and Shishkin 2018.
} 
R. Alonso and H. Rantakari. The art of brevity. working paper, 2013.

R. Aumann and S. Hart. Long cheap talk. Econometrica, 71(6):1619-1660, November 2003.

M. Backus and A. Little. I don’t know. Working Paper, 2018.

R. Bénabou and G. Laroque. Using priviledged information to manipulate markets: Insiders, gurus, and credibility. Quarterly Journal of Economics, 107(3):921-958, August 1992.

D. Bergemann and S. Morris. Information design: A unified perspective. Journal of Economic Literature, 57(1):44-95, 2019.

D. Bernhardt, C. Wan, and Z. Xiao. The reluctant analyst. Journal of Accounting Research, 54(4): 987-1040, 2016.

Y. Chen, M. Goltsman, J. Hörner, and G. Pavlov. Straight talk. working paper, 2017.

V. Crawford and J. Sobel. Strategic information transmission. Econometrica, 50(6):1431-1451, November 1982.

A. Dasgupta and A. Prat. Information aggregation in financial markets with career concerns. Journal of Economic Theory, 143(1):83-113, November 2008.

R. Deb, M. M. Pai, and M. Said. Evaluating strategic forecasters. American Economic Review, 108 (10):3057-3103, October 2018. doi: 10.1257/aer.20170299.

M. Dewatripont, I. Jewitt, and J. Tirole. The economics of career concerns, part i: Comparing information structures. Review of Economic Studies, 66(1):183-198, January 1999.

H. Di Pei. Reputation with strategic information disclosure. working paper, 2016.

M. Drugov and M. Troya-Martinez. Vague lies and lax standards of proof: On the law and economics of advice. Journal of Economics \&3 Management Strategy, 28(2):298-315, 2019.

W. Dziuda and C. Salas. Communication with detectable deceit. working paper, 2019.

J. Ely and J. Välimäki. Bad reputation. Quarterly Journal of Economics, 118(3):785-814, August 2003.

B. Ginzburg. A simple model of competitive testing. working paper, 2019.

J. R. Graham. Herding among investment newsletters: theory and evidence. Journal of Finance, 54(1):237-268, February 1999.

G. Gratton, R. Holden, and A. Kolotilin. When to drop a bombshell. Review of Economic Studies, 85(4):2139-2172, 2017.

S. Grenadier, A. Malenko, and N. Malenko. Timing decisions in organizations: Communication and authority in a dynamic environment. American Economic Review, 106(9):2552-2581, September 2016. 
I. Guttman. The timing of analysts' earnings forecasts. Accounting Review, 85(2):513-545, March 2010.

I. Guttman, I. Kremer, and A. Skrzypacz. Not only what but also when: A theory of dynamic voluntary disclosure. American Economic Review, 104(8):2400-2420, August 2014.

B. Holmström. Managerial incentive problems: A dynamic perspective. Review of Economic Studies, 66(1):169-182, January 1999.

H. Hong, J. D. Kubik, and A. Solomon. Security analysts' career concerns and herding of earnings forecasts. RAND Journal of Economics, 31(1):121-144, Spring 2000.

E. Kamenica and M. Gentzkow. Bayesian persuasion. American Economic Review, 101(6):2590$2615,2011$.

S. Keskek, S. Tse, and J. W. Tucker. Analyst information production and the timing of annual earnings forecasts. Review of Accounting Studies, 19(4):1504-1531, December 2014.

N. Klein and T. Mylovanov. Will truth out? an advisor's quest to appear competent. Journal of Mathematical Economics, 72:112-121, October 2017.

V. Krishna and J. Morgan. The art of conversation: Eliciting information from experts through multi-stage communication. Journal of Economic Theory, 117(2):147-179, August 2004.

O. A. Lamont. Macroeconomic forecasts and microeconomic forecasters. Journal of Economic Behavior \& Organization, 48(3):265-280, July 2002.

E. Lipnowski and D. Ravid. Cheap talk with transparent motives. working paper, 2019.

E. Lipnowski, D. Ravid, and D. Shishkin. Persuasion via weak institutions. working paper, 2018.

B. Mariano. Market power and reputational concerns in the ratings industry. Journal of Banking \& Finance, 36(6):1616-1626, June 2012.

S. Morris. Political correctness. Journal of Political Economy, 109(2):231-265, April 2001.

W. Olszewski. Calibration and expert testing. Handbook of Game Theory with Economic Applications, 4:949-984, 2015.

M. Ottaviani and P. N. Sørensen. Professional advice. Journal of Economic Theory, 126(1):120-142, January 2006a.

M. Ottaviani and P. N. Sørensen. The strategy of professional forecasting. Journal of Financial Economics, 81(2):441-466, August 2006b.

M. Ottaviani and P. N. Sørensen. Reputational cheap talk. RAND Journal of Economics, 37(1): 155-175, March 2006c.

A. Pavan, I. Segal, and J. Toikka. Dynamic mechanism design: A myersonian approach. Econometrica, 82(2):601-653, March 2014. 
F. Pavesi and M. Scotti. Good lies. working paper, 2017.

A. Prat. The wrong kind of transparency. American Economic Review, 95(3):862-877, June 2005.

C. Prendergast and L. Stole. Impetuous youngsters and jaded old-timers: Acquiring a reputation for learning. Journal of Political Economy, 104(6):1105-1134, December 1996.

D. Rodina. Information design and career concerns. working paper, 2017.

D. S. Scharfstein and J. C. Stein. Herd behavior and investment. American Economic Review, 80 (3):465-479, June 1990.

A. Smolin. Dynamic evaluation design. working paper, 2018.

J. Sobel. A theory of credibility. Review of Economic Studies, 52(4):557-573, October 1985.

B. Trueman. Analyst forecasts and herding behavior. Review of Financial Studies, 7(1):97-124, January 1994.

J. Zábojnik. On the efficiency of markets for managers. Economic Theory, 18(3):701-710, November 2001.

\section{Appendix}

Proof of Proposition 1. The proof is valid for all $\pi \in\left(\frac{1}{2}, 1\right]$. We show that $r^{E}(m, t)>0$ if and only if $r^{Q}(m, t)>0$ for any $(m, t)$ for any history with $b\left(h_{t}^{p}\right) \in(0,1)$. Together with the fact that $b_{0} \in(0,1)$, this will then mean that on equilibrium path we never arrive at a [non-terminal] history with $b\left(h_{t}^{p}\right) \in\{0,1\}$, hence the statement is true for all histories on equilibrium path.

Part 1: $r^{E}(m, t)>0 \Rightarrow r^{Q}(m, t)>0$. Suppose by contradiction that $r^{Q}(m, t)=0$. Then $b(m, t)=1$, meaning that $W_{t}^{Q}(m, t)$ attains maximum among all continuation payoffs (feasible or not). The initial assumption $r^{Q}(m, t)=0$ then means that either $W_{t}^{Q}(\varnothing)$, or $W_{t}^{Q}(m, s)$ for some $m$ and $s>t$ attain maximum, since one of these options should be more appealing to the quack than report $(m, t)$. These payoffs, however, cannot attain maximum, since $b_{t}<1$.

Part 2: $r^{Q}(m, t)>0 \Rightarrow r^{E}(m, t)>0$. Suppose that $r^{E}(m, t)=0$. Since $r^{Q}(m, t)>0$, we have $b(m, t)=0$, and hence $W_{t}^{Q}(m, t)$ attains minimum among all continuation payoffs. However, since belief about the forecaster's type is a martingale from the observer's point of view, we have either $b(-m, t)>0$ or $b_{t+1}>0$. Thus at least one of these strategies (reporting $-m$ or staying silent at $t$ ) strictly dominates the strategy of reporting $(m, t)$ for the quack, so $r^{Q}(m, t)=0$.

Similarly, one can show that $r^{Q}(G, s)+r^{Q}(B, s)=1$ if and only if $r^{E}(G, s)+r^{E}(B, s)=1$. Indeed, if for some $t$ we have $r^{E}(G, t)+r^{E}(B, t)=1$ and $r^{Q}(G, t)+r^{Q}(B, t)<1$, then not making a report by $t$ grants quack a continuation payoff of zero, while by martingale property of belief there exists $m \in\{G, B\}$ such that $b(m, t)>0$, and therefore report $(m, t)$ dominates the strategy of staying silent. Similarly, if $r^{E}(G, t)+r^{E}(B, t)<1$ and $r^{Q}(G, t)+r^{Q}(B, t)=1$, then not making a report by $t$ yields the maximal continuation payoff, while again by the martingale property making at least some report gives strictly less in expectation. 
Before we proceed, it is useful to introduce some new pieces of notation which come in handy for further proofs. The expert's report probabilities can be rewritten as

$$
\begin{aligned}
r^{E}(m, t)= & \mathbb{E}_{\eta}\left[r_{\eta}^{E}(m, t)\right]=\frac{\tilde{p}_{0} \cdot z_{t, G} \cdot r_{G}^{E}(m, t)+\left(1-\tilde{p}_{0}\right) \cdot z_{t, B} \cdot r_{B}^{E}(m, t)+z_{t, \varnothing} \cdot r_{\varnothing}^{E}(m, t)}{\tilde{p}_{0} \cdot z_{t, G}+\left(1-\tilde{p}_{0}\right) \cdot z_{t, B}+z_{t, \varnothing}}, \\
& \mathbb{E}_{\eta}\left[r_{\eta}^{E}(m, t) \mid \omega\right]=\frac{\pi \cdot z_{t, \omega} \cdot r_{\omega}^{E}(m, t)+(1-\pi) \cdot z_{t,-\omega} \cdot r_{-\omega}^{E}(m, t)+z_{t, \varnothing} \cdot r_{\varnothing}^{E}(m, t)}{\pi \cdot z_{t, \omega}+(1-\pi) \cdot z_{t,-\omega}+z_{t, \varnothing}},
\end{aligned}
$$

where $\tilde{p}_{0}=p_{0} \pi+\left(1-p_{0}\right)(1-\pi)$, and $z_{t, \eta}=P\left\{\eta_{t}=\eta, \mu_{t-1}=\varnothing \mid \eta^{*}=\eta\right\}$ for $\eta \in\{\varnothing, G, B\}$. I.e., $z_{t, \eta}$ is the probability that the expert has information $\eta$ at time $t$ and has not made a report prior to $t$, conditional on expert's signal realization being $\eta^{*}=\eta$ (or unconditional if $\eta=\varnothing$ ). It can be expressed recursively as

$$
\begin{aligned}
& z_{t, \eta}=z_{t-1, \eta} \cdot\left(1-\sum_{m} r_{\eta}^{E}(m, t-1)\right)+z_{t-1, \varnothing} \cdot \lambda(t) \cdot\left(1-\sum_{m} r_{\varnothing}^{E}(m, t-1)\right), \\
& z_{t, \varnothing}=z_{t-1, \varnothing} \cdot(1-\lambda(t)) \cdot\left(1-\sum_{m} r_{\varnothing}^{E}(m, t-1)\right)
\end{aligned}
$$

with $z_{0, G}=z_{0, B}=0$ and $z_{0, \varnothing}=1$. In any symmetric equilibrium we have $z_{t, G}=z_{t, B} \equiv z_{t}$, so the expectations above transform into

$$
\begin{aligned}
r^{E}(m, t)=\mathbb{E}_{\eta}\left[r_{\eta}^{E}(m, t)\right] & =z_{t}\left(\tilde{p}_{0} r_{G}^{E}(m, t)+\left(1-\tilde{p}_{0}\right) r_{B}^{E}(m, t)\right)+\left(1-z_{t}\right) r_{\varnothing}^{E}(m, t), \\
\mathbb{E}_{\eta}\left[r_{\eta}^{E}(m, t) \mid \omega\right] & =z_{t}\left(\pi r_{\omega}^{E}(m, t)+(1-\pi) r_{-\omega}^{E}(m, t)\right)+\left(1-z_{t}\right) r_{\varnothing}^{E}(m, t),
\end{aligned}
$$

where $z_{t}=\frac{z_{t}}{z_{t}+z_{t, \varnothing}}$ and $1-z_{t}=\frac{z_{t, \varnothing}}{z_{t}+z_{t, \varnothing}}$.

Given the strategies, we also define the likelihood ratio of reports as

$$
g(m, t):=\ln (\beta(m, t))-\ln \left(\beta_{t-1}\right)=\ln \left(\frac{r^{E}(m, t)}{r^{Q}(m, t)}\right)
$$

with $\pm \infty$ being admissible values. This ratio summarizes the information about the forecaster's type contained in report $(m, t)$.

Proof of Proposition 2. The proof is valid for all $\pi \in\left(\frac{1}{2}, 1\right]$. We begin with a useful observation:

$$
W_{t, \varnothing}^{E}(m, t)=W_{t}^{Q}(m, t) \text { for } m \in\{G, B\}
$$

If the expert reports $(m, t)$ before observing a private signal, his continuation payoff coincides with that of the quack, since they possess the same private information at any such history.

Note further that the existence of a Godwin point $\bar{t}=\min \left\{t \in \mathcal{T} \mid V_{t, \varnothing}^{E}=V_{t}^{Q}\right\}$ is trivial since the required equality is always satisfied for the last point of $\mathcal{S}$. To see this, observe that any report $(m, t)$ for $t>t_{|\mathcal{S}|}$ yields zero reputation for the rest of the game due to assumption (OP), and is therefore weakly dominated for any type of the forecaster by staying silent. At the same time, staying silent yields the same time- $t$ expected payoff to the uninformed (as of time $t$ ) expert as it does to the quack, since they have the same information. This together with 15 gives the result.

Most of the remaining proof is devoted to showing that $V_{t, \varnothing}^{E}=V_{t}^{Q}$ for some $t$ implies babbling in all further times. This is established in a series of claims. The second part of the proposition is then easily shown by contradiction.

As a starting point, we show that $W_{t, \eta}^{E}(m, t)=W_{t}^{Q}(m, t)$ for any $m \in\{G, B\}$, any $\eta \in\{G, B\}$ and any 
$t \in \mathcal{S}^{m}$ such that $t>\bar{t}$. Suppose the converse - there exist $m, t$ and $\eta$ such that $W_{t, \eta}^{E}(m, t) \neq W_{t}^{Q}(m, t)$. Then (ML) and (SY) imply that there can be three cases:

Case 1: $r_{G}^{E}(G, t)=r_{B}^{E}(B, t) \geqslant r_{G}^{E}(B, t)=r_{B}^{E}(G, t)>0$.

In this case $W_{t, G}^{E}(G, t)=W_{t, G}^{E}(B, t)$ and, by Proposition $1, V_{t}^{Q}=W_{t}^{Q}(G, t)=W_{t}^{Q}(B, t)$. Therefore, $W_{t, G}^{E}(G, t)-W_{t}^{Q}(G, t)=W_{t, G}^{E}(B, t)-W_{t}^{Q}(B, t)$, which reduces to the equality of differences in terminal reputation:

$$
w^{c}\left(\beta^{G}(G, t)\right)-w^{c}\left(\beta^{B}(G, t)\right)=-w^{c}\left(\beta^{B}(B, t)\right)+w^{c}\left(\beta^{G}(B, t)\right) .
$$

If $r_{G}^{E}(G, t)>r_{B}^{E}(G, t)$ then, by (14) and the expression for $b^{\omega}(m, t)$, the LHS of (16) is weakly positive. However, due to (SY) we then have that $r_{G}^{E}(B, t)<r_{B}^{E}(B, t)$, so the RHS is weakly negative. The converse also holds, which leaves us with the conclusion that for 16 to be satisfied, its both sides must be equal to zero. Therefore, $\beta^{G}(m, t)=\beta^{B}(m, t)$ for any $m \in\{G, B\}$, which implies $W_{t, \eta}^{E}(m, t)=W_{t}^{Q}(m, t)=V_{t}^{Q}$ for any $\eta \in\{G, B\}$ and any $m \in\{G, B\},-$ a contradiction.

Case 2: $r_{G}^{E}(G, t)=r_{B}^{E}(B, t)>0=r_{G}^{E}(B, t)=r_{B}^{E}(G, t)$.

As $r_{G}^{E}(G, t)>r_{B}^{E}(G, t)$ and $r_{B}^{E}(B, t)>r_{B}^{E}(G, t)$, we have that $V_{t, G}^{E}=W_{t, G}^{E}(G, t)>W_{t}^{Q}(G, t)=V_{t}^{Q}$ and, analogously, $V_{t, B}^{E}>V_{t}^{Q}$. Next, note that $V_{\bar{t}, \varnothing}^{E}$ is, for all $t>\bar{t}$, bounded below by

$$
\sum_{s=\bar{t}}^{t-1} w\left(\beta_{s}\right)+P\left\{t^{*} \leqslant t \mid t^{*}>\bar{t}\right\} \cdot\left(\tilde{p}_{0} \cdot V_{t, G}^{E}+\left(1-\tilde{p}_{0}\right) \cdot V_{t, B}^{E}\right)+P\left\{t^{*}>t \mid t^{*}>\bar{t}\right\} \cdot V_{t, \varnothing}^{E},
$$

which is the value of not making a report from $\bar{t}$ until at least $t$. By 15 we have $V_{t, \varnothing}^{E} \geqslant W_{t, \varnothing}^{E}(m, t)=$ $W_{t}^{Q}(m, t)$. Second, we have shown that $V_{t, \eta}^{E}>V_{t}^{Q}$. Therefore,

$$
V_{\bar{t}, \varnothing}^{E}>\sum_{s=\bar{t}}^{t-1} w\left(\beta_{s}\right)+W_{t}^{Q}(m, t)=V_{\bar{t}}^{Q}
$$

which gives us a contradiction with the definition of $\bar{t}$.

Case 3: $r_{G}^{E}(G, t)=r_{B}^{E}(B, t)=r_{G}^{E}(B, t)=r_{B}^{E}(G, t)=0$ and $r_{\varnothing}^{E}(m, t)>0$ for some $m$. $r_{G}^{E}(G, t)=r_{G}^{E}(B, t)=r_{B}^{E}(G, t)=r_{B}^{E}(B, t)$ automatically implies $W_{t, \eta}^{E}(m, t)=W_{t}^{Q}(m, t)$ for any $\eta \in\{G, B\}$ and any $m \in\{G, B\}$, which gives us a contradiction with the initial assumption.

Next we show that $W_{t, \eta}^{E}(m, t)=W_{t}^{Q}(m, t)$ for all $\eta, m \in\{G, B\}$ with $t \in \mathcal{S}^{m}$ implies that report $(m, t)$ is babbling. Without loss of generality assume $\eta=G$. Expanding the equality, we see that

$$
0=W_{t, G}^{E}(m, t)-W_{t}^{Q}(m, t)=\frac{p_{0} \cdot\left(1-p_{0}\right) \cdot(2 \pi-1)}{\tilde{p}_{0}} \cdot\left(w^{c}\left(\beta^{G}(m, t)\right)-w^{c}\left(\beta^{B}(m, t)\right)\right),
$$

and therefore $\beta^{G}(m, t)=\beta^{B}(m, t)$. It further implies that 7 reduces to 10 . In other words, it follows that reputation should not be affected by the revelation of state after any time- $t$ report.

To conclude that only babbling is possible after $\bar{t}$ we are left to show that $(9)$ holds for all $(m, s)$ with $s>\bar{t}$. Condition $(9)$ is equivalent to $g(m, s)=0$. Three cases are possible (since we have shown in the proof of Proposition 1 that $r^{\gamma}(G, s)+r^{\gamma}(B, s)=1$ cannot be the case for exactly one $\left.\gamma\right)$.

Case 1: $s=\max \{t \in \mathcal{S} \mid t>\bar{t}\}$ and $r^{\gamma}(G, s)+r^{\gamma}(B, s)=1$ for any $\gamma \in\{S, Q\}$.

If $m$ is the only report made at $s$ then $r_{\eta}^{\gamma}(m, s)=1$ for all $\gamma, \eta$, which implies $g(m, s)=0$. If both reports are made on path at $s$, then by the same $\operatorname{logic} r_{\eta}^{\gamma}(G, s)+r_{\eta}^{\gamma}(B, s)=1$, and if $g(m, s) \neq 0$ 
for some $m$ then the report with higher $g(m, s)$ is strictly preferred by either forecaster, contradicting that both reports occur on path.

Case 2: $s=\max \{t \in \mathcal{S} \mid t>\bar{t}\}$ and $r^{\gamma}(G, s)+r^{\gamma}(B, s)<1$ for $\gamma \in\{S, Q\}$.

If $m$ is the only report made at $s$ and $g(m, s) \neq 0$ then a quack has strict preference between report $(m, s)$ and staying silent at $s$ (because in either case he gets a degenerate lottery at $T$, since 10 is satisfied). This strict preference cannot occur in equilibrium, thus $g(m, s)=0$. If both reports are made on path at $s$ then we can combine the two indifference arguments above to obtain that $g(G, s)=g(B, s)$ and, consequently, $g(m, s)=0$ for all $m \in\{G, B\}$.

Case 3: $s<\max \{t \in \mathcal{S} \mid t>\bar{t}\}$.

From the previous case we know that $g(m, s)=0$ for any on-path $m$ at $s=\max \{t \in \mathcal{S} \mid t>\bar{t}\}$. We can iterate backwards from there as follows. If $s-1 \in \mathcal{S}$ then a quack should be indifferent between making an on-path report at $s-1$ and at $s$, which can only happen if $g(m, s-1)=0$, because $g(m, s)=0$ and 100 is satisfied for both reports. Iterating backwards we establish the claim for all $t>\bar{t}$. If some of these periods are not in $\mathcal{S}$ then they can be skipped because beliefs do not change at such periods.

All of the above proves that only babbling is possible after the Godwin point.

We are left to show the second part of the proposition. First, suppose there exist $m$ and $t<\bar{t}$ such that $r_{\varnothing}^{E}(m, t)>0$. Then $V_{t, \varnothing}^{E}=W_{t, \varnothing}^{E}(m, t)=W_{t}^{Q}(m, t)=V_{t}^{Q}$, where the second equality follows from 15. Thus $t \geqslant \bar{t}$ by definition of $\bar{t}-$ a contradiction.

Now suppose there exists $t \leqslant \bar{t}$ such that $r_{\eta}^{E}(-\eta, t)>0$ for some $\eta \in\{G, B\}$. As shown before, it implies $V_{t, \eta}^{E}=W_{t, \eta}^{E}(m, t)=W_{t}^{Q}(m, t)=V_{t}^{Q}$ for all $\eta, m \in\{G, B\}$. Suppose first that $t=\bar{t}$. Then as $r_{\varnothing}^{E}\left(m, t_{|\mathcal{S}|-1}\right)=0$ for $m \in\{G, B\}$, we have

$$
V_{t_{|\mathcal{S}|-1}, \varnothing}^{E}=w\left(\beta_{t_{|\mathcal{S}|-1}}\right)+P\left\{t^{*} \leqslant \bar{t} \mid t^{*}>t_{|\mathcal{S}|-1}\right\} \cdot\left(\tilde{p}_{0} \cdot V_{\bar{t}, G}^{E}+\left(1-\tilde{p}_{0}\right) \cdot V_{\bar{t}, B}^{E}\right)+P\left\{t^{*}>\bar{t} \mid t^{*}>t_{|\mathcal{S}|-1}\right\} \cdot V_{\bar{t}, \varnothing}^{E} .
$$

As $V_{\bar{t}, \varnothing}^{E}=V_{\bar{t}}^{Q}$ and $V_{\bar{t}, \eta}^{E}=V_{\bar{t}}^{Q}$, the above expression reduces to $V_{t_{|\mathcal{S}|-1}, \varnothing}^{E}=w\left(\beta_{t_{|\mathcal{S}|-1}}\right)+V_{\bar{t}}^{Q}=V_{t_{|\mathcal{S}|-1}}^{Q}$, which constitutes a contradiction with the definition of the Godwin point. One can similarly show that $r_{\eta}^{E}(\eta, \bar{t})>0$, as otherwise $V_{\bar{t}, \eta}^{E}=W_{\bar{t}, \eta}^{E}(m, \bar{t})=W_{t}^{Q}(m, \bar{t})=V_{\bar{t}}^{Q}$ for all $\eta \in\{G, B\}$ and all $m \in\{G, B\}$, which leads to the same contradiction with the definition of the Godwin point as above. Finally, if $t<\bar{t}$ then, as $r_{\eta}^{E}(-\eta, \bar{t})=0$ and $r_{\eta}^{E}(\eta, \bar{t})>0$ imply $V_{\bar{t}, \eta}^{E}>V_{\bar{t}}^{Q}$, a competent forecaster who has received a signal by period $t$ can postpone his report until $\bar{t}$ and receive strictly more than the quack which contradicts $V_{t, \eta}^{E}=V_{t}^{Q}$ implied by $r_{\eta}^{E}(-\eta, t)>0$.

We are left to show that the expert never wants to conceal his private signal. Assume $r_{\eta}^{E}(\eta, \bar{t})<1$. Then thet expert must weakly prefer to conceal his private signal than to report it. In the first case the expert receives exactly $V_{\bar{t}}^{Q}$, while in the latter he gets $V_{\bar{t}, \eta}^{E}>V_{\bar{t}}^{Q}$,- a contradiction.

Proof of Proposition 3 The proof is valid for all $\pi \in\left(\frac{1}{2}, 1\right]$. Let $\left\{r_{\eta}^{\gamma}(m, t)\right\}$ be an equilibrium strategy profile. Consider a new strategy profile $\left\{\tilde{r}_{\eta}^{\gamma}(m, t)\right\}$ such that $\tilde{r}_{t, \eta}^{E}(m, t)=r_{t, \eta}^{E}(m, t), \tilde{r}_{t}^{Q}(m, t)=r_{t}^{Q}(m, t)$ for all $t \leqslant \bar{t}$ and $\tilde{r}_{t, \eta}^{E}(m, t)=\tilde{r}_{t}^{Q}(m, t)=0$ for all $t>\bar{t}$. As strategies coincide on $\tilde{\mathcal{S}}$ and all reports $(m, t)$ with $t>\bar{t}$ are babbling in the original equilibrium, the following are true:

1. beliefs $b(m, t)$ and $b^{\omega}(m, t)$ induced by the two strategy profiles coincide for all $\omega, m \in\{G, B\}, t \in \tilde{\mathcal{S}}$;

2. belief sequences $b_{t}$ induced by the two strategy profiles coincide for all $t \in \mathcal{T}$.

The latter statement also exploits the fact that $\mathcal{S} \backslash \tilde{\mathcal{S}}$ is nonempty (otherwise the proposition statement trivially holds), so it must be that $r^{Q}(G, \bar{t})+r^{Q}(B, \bar{t})<1$ and $r^{E}(G, \bar{t})+r^{E}(B, \bar{t})<1$. 
The first statement above implies that any report $(m, t)$ with $t \leqslant \bar{t}$ yields the same payoff under either strategy profile. The second statement states that reporting nothing in any period yields the same payoffs as well. Strategy of reporting nothing yields the same payoff under $\left\{\tilde{r}_{\eta}^{\gamma}(m, t)\right\}$ as any report $(m, t)$ with $t>\bar{t}$ under $\left\{r_{\eta}^{\gamma}(m, t)\right\}$, since all such reports are babbling. Finally, any report $(m, t)$ with $t \notin \mathcal{S}$ yields the same payoff under either strategy profile due to $(\mathrm{OP})$.

Everything said above directly implies that if $r_{\eta}^{\gamma}(m, t)$ is a best response for type- $\gamma$ forecaster to strategy profile $\left\{r_{\eta}^{\gamma}(m, t)\right\}$ then $\tilde{r}_{\eta}^{\gamma}(m, t)$ is a best response for him to strategy profile $\left\{\tilde{r}_{\eta}^{\gamma}(m, t)\right\}$ and yields the same payoff.

\section{Proof of the Main Result}

Before proceeding to the proof of Theorem 1, we provide some expressions for belief updating that will be useful in further proofs. Using Proposition 2 and the notion of $z_{t}$ introduced earlier in this Appendix, we can rewrite the expressions for (4) and (6) in a more explicit form. Proposition 2 implies that for all $t<\bar{t}$ we have $r_{G}^{E}(B, t)=r_{B}^{E}(G, t)=0$. Therefore, 13 and $(14)$ together imply that for all $t \in \mathcal{S}$ we have

$$
\begin{aligned}
& \beta(G, t)=\beta_{t-1} \cdot \frac{r^{E}(G, t)}{r^{Q}(G, t)}=\beta_{t-1} \cdot \frac{\tilde{p}_{0} z_{t} r_{G}^{E}(G, t)+\left(1-z_{t}\right) r_{\varnothing}^{E}(G, t)}{r^{Q}(G, t)} \\
& \beta(B, t)=\beta_{t-1} \cdot \frac{r^{E}(B, t)}{r^{Q}(B, t)}=\beta_{t-1} \cdot \frac{\left(1-\tilde{p}_{0}\right) z_{t} r_{B}^{E}(B, t)+\left(1-z_{t}\right) r_{\varnothing}^{E}(B, t)}{r^{Q}(B, t)},
\end{aligned}
$$

as well as

$$
\begin{aligned}
& \beta^{G}(G, t)=\beta_{t-1} \cdot \frac{\mathbb{E}_{\eta}\left[r_{\eta}^{E}(G, t) \mid G\right]}{r^{Q}(G, t)}=\beta_{t-1} \cdot \frac{\pi z_{t} r_{G}^{E}(G, t)+\left(1-z_{t}\right) r_{\varnothing}^{E}(G, t)}{r^{Q}(G, t)}, \\
& \beta^{B}(B, t)=\beta_{t-1} \cdot \frac{\mathbb{E}_{\eta}\left[r_{\eta}^{E}(B, t) \mid B\right]}{r^{Q}(B, t)}=\beta_{t-1} \cdot \frac{\pi z_{t} r_{B}^{E}(B, t)+\left(1-z_{t}\right) r_{\varnothing}^{E}(B, t)}{r^{Q}(B, t)}, \\
& \beta^{B}(G, t)=\beta_{t-1} \cdot \frac{\mathbb{E}_{\eta}\left[r_{\eta}^{E}(G, t) \mid B\right]}{r^{Q}(G, t)}=\beta_{t-1} \cdot \frac{(1-\pi) z_{t} r_{G}^{E}(G, t)+\left(1-z_{t}\right) r_{\varnothing}^{E}(G, t)}{r^{Q}(G, t)}, \\
& \beta^{G}(B, t)=\beta_{t-1} \cdot \frac{\mathbb{E}_{\eta}\left[r_{\eta}^{E}(B, t) \mid G\right]}{r^{Q}(B, t)}=\beta_{t-1} \cdot \frac{(1-\pi) z_{t} r_{B}^{E}(B, t)+\left(1-z_{t}\right) r_{\varnothing}^{E}(B, t)}{r^{Q}(B, t)},
\end{aligned}
$$

and It is also worth remembering that $r_{\varnothing}^{E}(m, t)=0$ for any $t<\bar{t}$.

In case no report was made in period $t<\bar{t}$, the belief is updated as

$$
\beta_{t}=\beta_{t-1} \cdot \frac{1-r^{E}(G, t)-r^{E}(B, t)}{1-r^{Q}(G, t)-r^{Q}(B, t)}=\beta_{t-1} \cdot \frac{1-z_{t} r_{G}^{E}(G, t)}{1-r^{Q}(G, t)-r^{Q}(B, t)},
$$

while the analogous expression for $t=\bar{t}$ is given by

$$
\beta_{\bar{t}}=\beta_{t_{|\mathcal{S}|-1}} \cdot \frac{1-r^{E}(G, \bar{t})-r^{E}(B, \bar{t})}{1-r^{Q}(G, \bar{t})-r^{Q}(B, \bar{t})}=\beta_{t_{|\mathcal{S}|-1}} \cdot \frac{\left(1-z_{\bar{t}}\right) \cdot\left(1-r_{\varnothing}^{E}(G, \bar{t})-r_{\varnothing}^{E}(B, \bar{t})\right)}{1-r^{Q}(G, \bar{t})-r^{Q}(B, \bar{t})}
$$

In 190 we use the fact that $r_{G}^{E}(G, t)=r_{B}^{E}(B, t)$ due to $(\mathrm{SY})$, and in 20 we use that $r_{G}^{E}(G, \bar{t})=r_{B}^{E}(B, \bar{t})=1$ by Proposition 2 .

What follows is the proof of the main result, Theorem 1. To avoid duplicating the arguments, we merge it with the proof of Propositions 7 and 9

Proof of Theorem 1 and Propositions 7 and 9. First, recall that Propositions 1, 2 and 3 are true for all $\pi \in\left(\frac{1}{2}, 1\right]$, and so can be employed in this proof. Further, note that in all babbling periods $t$ we have $i(m, t)=0, b(m, t)=b_{t-1}$ for $m \in\{G, B\}$, and $b_{t}$ stays on a constant level. Together with Propositions 2 
and 3 this means that it is enough to show the statement of the Theorem for informative equilibria. The proof is separated into several steps.

Step 1. We start by showing that $\Delta w_{\eta}(m, t)$, which is defined as

$$
\Delta w_{\eta}(m, t):=w^{c}\left(\beta^{\eta}(m, t)\right)-w^{c}\left(\beta^{-\eta}(m, t)\right),
$$

is a weakly decreasing function of $t$ on $\mathcal{S}$ given $m=\eta$ (note that $\Delta w_{G}(m, t)=-\Delta w_{B}(m, t)$ ). Suppose the expert has private information $\eta=G$ at time $t$, but has not yet made any report. He chooses a report $(m, \tau)$ with $\tau \geqslant t$ which maximizes $W_{t, G}^{E}(m, \tau)$, where "making no report" is also an available option. Expanding $W_{t, G}^{E}(m, \tau)$, we get the following expression:

$$
\sum_{s=t}^{\tau-1} w\left(\beta_{s}\right)+\sum_{s=\tau}^{T-1} w(\beta(m, \tau))+\frac{\pi p_{0}}{\tilde{p}_{0}} w^{c}\left(\beta^{G}(m, \tau)\right)+\left(1-\frac{\pi p_{0}}{\tilde{p}_{0}}\right) w^{c}\left(\beta^{B}(m, \tau)\right)
$$

where $\tilde{p}_{0}=p_{0} \pi+\left(1-p_{0}\right)(1-\pi)$.

A quack is indifferent between all such reports at time $t$. His continuation value $W_{t}^{Q}(m, \tau)$ can similarly be written as

$$
\sum_{s=t}^{\tau-1} w\left(\beta_{s}\right)+\sum_{s=\tau}^{T-1} w(\beta(m, \tau))+p_{0} w^{c}\left(\beta^{G}(m, \tau)\right)+\left(1-p_{0}\right) w^{c}\left(\beta^{B}(m, \tau)\right)
$$

Given that the latter expression is constant over all $(m, \tau)$, the optimization problem of an expert with $\eta=G$ becomes equivalent to maximizing the difference $\Delta w_{G}(m, \tau)$ over all $\tau \in\{\mathcal{S} \mid \tau \geqslant t\}$ and $m \in\{G, B\}$.

Similarly, an expert who has observed signal $B$ chooses report $(m, \tau)$ which maximizes $\Delta w_{B}(m, \tau)$. Propositions 1, 2 and 3 imply that $\mathcal{S}=\left\{t \in \mathcal{T} \mid r_{\eta}^{E}(\eta, t)>0\right\}$ for any $\eta \in\{G, B\}$, so since $t \in \mathcal{S}$, it must be that $(G, t)$ maximizes $\Delta w_{G}(m, \tau)$ and $(B, t)$ maximizes $\Delta w_{B}(m, \tau)$ across all $(m, \tau)$ with $\tau \in\{\mathcal{S} \mid \tau \geqslant t\}$. Therefore, $\Delta w_{\eta}(\eta, t)$ must be a weakly decreasing function of $t$ on $\mathcal{S}$.

Step 2. The second step of the proof consists in showing that for $\eta \in\{G, B\}, \Delta w_{\eta}(\eta, t)$ is weakly decreasing on $\mathcal{S} \backslash\{\bar{t}\}$ if and only if $\beta^{\eta}(\eta, t)$ is weakly decreasing on $\mathcal{S} \backslash\{\bar{t}\}$ (if an equilibrium is reticent then $\Delta w_{\eta}(\eta, t)$ is weakly decreasing on $\mathcal{S}$ if and only if $\beta^{\eta}(\eta, t)$ is weakly decreasing on $\left.\mathcal{S}\right)$. We demonstrate it for all cases stated in Theorem 1, Proposition 7 and Proposition 9 separately.

Case 1: $\pi=1$

This case is obvious, as then $w\left(\beta^{-\eta}(\eta, t)\right)=0$ for any $\eta \in\{G, B\}$, and $w(\cdot)$ is a strictly increasing function.

Case 2: $w^{c}(\cdot)$ is convex and $\pi<1$.

Note that since $r_{\varnothing}^{E}(m, t)=0$ for all $t \in \mathcal{S} \backslash\{\bar{t}\}$ (or for all $t \in \mathcal{S}$ if an equilibrium is reticent) from (18) we have

$$
\beta^{-\eta}(\eta, t)=\frac{1-\pi}{\pi} \cdot \beta^{\eta}(\eta, t),
$$

where $\frac{1-\pi}{\pi} \in(0,1)$ because $\pi>\frac{1}{2}$. Take any $\tau_{1}>\tau_{2}$ with $\tau_{1}, \tau_{2} \in \mathcal{S}$. Then if $\beta^{\eta}\left(\eta, \tau_{1}\right)=x_{1}>x_{2}=$ $\beta^{\eta}\left(\eta, \tau_{2}\right)$ we have

$$
w^{c}\left(x_{1}\right)-w^{c}\left(\frac{1-\pi}{\pi} x_{1}\right) \geqslant w^{c}\left(x_{2}\right)-w^{c}\left(x_{2}-x_{1}+\frac{1-\pi}{\pi} x_{1}\right)>w^{c}\left(x_{2}\right)-w^{c}\left(\frac{1-\pi}{\pi} x_{2}\right),
$$

where the first inequality follows from convexity of $w(\beta)$, and the second is valid because $w(\beta)$ is 
strictly increasing.

Case 3: $\pi>\frac{\bar{d}}{\underline{d}+\bar{d}}$ and $\frac{d w^{c}(\beta)}{d \beta} \in[\underline{d}, \bar{d}]$.

Similarly to the previous case take any $\tau_{1}, \tau_{2} \in \mathcal{S}$ and let $x_{1}:=\beta^{\eta}\left(\eta, \tau_{1}\right), x_{2}:=\beta^{\eta}\left(\eta, \tau_{2}\right)$. Suppose $w^{c}\left(x_{2}\right)-w^{c}\left(\frac{1-\pi}{\pi} x_{2}\right)>w^{c}\left(x_{1}\right)-w^{c}\left(\frac{1-\pi}{\pi} x_{1}\right)$. Then

$$
\begin{aligned}
0 & <\left(w^{c}\left(x_{2}\right)-w^{c}\left(\frac{1-\pi}{\pi} x_{2}\right)\right)-\left(w^{c}\left(x_{1}\right)-w^{c}\left(\frac{1-\pi}{\pi} x_{1}\right)\right)< \\
& <\bar{d} \cdot\left(x_{2}-x_{1}\right)-\underline{d} \cdot\left(\frac{1-\pi}{\pi} x_{2}-\frac{1-\pi}{\pi} x_{1}\right)<\left(x_{2}-x_{1}\right) \cdot\left(\bar{d}-\underline{d} \cdot \frac{1-\pi}{\pi}\right) .
\end{aligned}
$$

As $\left(\bar{d}-\underline{d} \cdot \frac{1-\pi}{\pi}\right)>0$, we must have $x_{2}>x_{1}$.

Conversely, if $x_{2}>x_{1}$ then

$$
0<\left(x_{2}-x_{1}\right) \cdot\left(\underline{d}-\bar{d} \cdot \frac{1-\pi}{\pi}\right)<\left(w^{c}\left(x_{2}\right)-w^{c}\left(\frac{1-\pi}{\pi} x_{2}\right)\right)-\left(w^{c}\left(x_{1}\right)-w^{c}\left(\frac{1-\pi}{\pi} x_{1}\right)\right)
$$

which grants the result.

Step 3. We next show that whenever $|\mathcal{S}| \geqslant 3$ and an equilibrium on $\mathcal{S}$ exists, it must be that $b_{t_{1}} \geqslant b\left(m, t_{1}\right)$ for any $m \in\{G, B\}$ (alternatively, if equilibrium is reticent, then this claim is valid for any $\mathcal{S}$ with $|\mathcal{S}| \geqslant 2)$. Assume there exists $m \in\{G, B\}$ such that $b_{t_{1}}<b\left(m, t_{1}\right)$. Quack's value from report $m$ at $t_{1}$ and $t_{2}$ are equal to

$$
\begin{aligned}
& W_{t_{1}}^{Q}\left(m, t_{1}\right)=\left(T-t_{1}\right) \cdot w\left(\beta\left(m, t_{1}\right)\right)+p_{0} w^{c}\left(\beta^{G}\left(m, t_{1}\right)\right)+\left(1-p_{0}\right) w^{c}\left(\beta^{B}\left(m, t_{1}\right)\right) \\
& W_{t_{1}}^{Q}\left(m, t_{2}\right)=\left(t_{2}-t_{1}\right) \cdot w\left(\beta_{t_{1}}\right)+\left(T-t_{2}\right) \cdot w\left(\beta\left(m, t_{2}\right)\right)+p_{0} w^{c}\left(\beta^{G}\left(m, t_{2}\right)\right)+\left(1-p_{0}\right) w^{c}\left(\beta^{B}\left(m, t_{2}\right)\right)
\end{aligned}
$$

As $w(\cdot)$ is strictly increasing, and $b_{t_{1}}<b\left(m, t_{1}\right), W_{t_{1}}^{Q}\left(m, t_{1}\right)=W_{t_{1}}^{Q}\left(m, t_{2}\right)$ implies

$$
\begin{aligned}
& \left(T-t_{2}\right) \cdot w\left(\beta\left(m, t_{1}\right)\right)+p_{0} w^{c}\left(\beta^{G}\left(m, t_{1}\right)\right)+\left(1-p_{0}\right) w^{c}\left(\beta^{B}\left(m, t_{1}\right)\right)< \\
& \left(T-t_{2}\right) \cdot w\left(\beta\left(m, t_{2}\right)\right)+p_{0} w^{c}\left(\beta^{G}\left(m, t_{2}\right)\right)+\left(1-p_{0}\right) w^{c}\left(\beta^{B}\left(m, t_{2}\right)\right) .
\end{aligned}
$$

Consequently, it must be that either $\beta\left(m, t_{1}\right)<\beta\left(m, t_{2}\right)$, or $\beta^{m}\left(m, t_{1}\right)<\beta^{m}\left(m, t_{2}\right)$ or $\beta^{-m}\left(m, t_{1}\right)<$ $\beta^{-m}\left(m, t_{2}\right)$. However, (17) and (18) imply that both $\beta^{G}(m, t)$ and $\beta^{B}(m, t)$ differ from $\beta(m, t)$ by a constant factor for any $t \in \mathcal{S} \backslash\{\bar{t}\}$ ( since $r_{\varnothing}^{E}(m, t)=0$ ), so the three inequalities are equivalent. Therefore, $\beta^{m}\left(m, t_{1}\right)<\beta^{m}\left(m, t_{2}\right)$, which contradicts $\beta^{m}(m, t)$ being decreasing on $\mathcal{S} \backslash\{\bar{t}\}$. In reticent equilibria $r_{\varnothing}^{E}(m, \bar{t})=0$, therefore the claim extends to $\bar{t}$ as well.

Step 4. We finally show how the claim in the theorem can be obtained from the previous steps. We have shown that $b_{t_{1}} \geqslant b\left(m, t_{1}\right)$ for any $m \in\{G, B\}$. Consequently, as $b_{t}$ is a martingale, we have that $b_{t_{1}} \geqslant b_{0}$ and $b\left(m, t_{1}\right) \leqslant b_{0}$ for at least one $m \in\{G, B\}$. As $b\left(m, t_{1}\right) \leqslant b_{t_{1}}$ for $m \in\{G, B\}$, we must have that either $b\left(m, t_{2}\right) \leqslant b\left(m, t_{1}\right)$, or $b^{m}\left(m, t_{2}\right) \leqslant b^{m}\left(m, t_{1}\right)$ or $b^{-m}\left(m, t_{2}\right) \leqslant b^{-m}\left(m, t_{1}\right)$ to make the quack indifferent between reports $\left(m, t_{1}\right)$ and $\left(m, t_{2}\right)$. Again, 17) and 18 imply that all three inequalities are equivalent, so all three have to hold. The fact that $b_{t}$ is a martingale together with resulting inequalities $b_{t_{1}} \geqslant b\left(m, t_{1}\right) \geqslant b\left(m, t_{2}\right)$ for $m \in\{G, B\}$ imply $b_{t_{2}} \geqslant b_{t_{1}}$. Iterating this argument further, we achieve that $b(m, t) \leqslant b_{t}$ and $b_{t}$ is increasing in $t$ on $\mathcal{S} \backslash\{\bar{t}\}$ (on whole $\mathcal{S}$ if equilibrium is reticent).

The above proves the second and the third parts of Theorem 1 and Propositions 7 and $9{ }^{29}$ It remains to show the first part. Note that, by the same inductive reasoning as above, if $b_{t_{1}}>b\left(m, t_{1}\right)$ then

\footnotetext{
${ }^{29}$ The statement that $b_{t}$ is constant on $\mathcal{T} \backslash \mathcal{S}$ follows trivially from 4 .
} 
$b_{t_{|\mathcal{S}|-1}}>b\left(m, t_{|\mathcal{S}|-1}\right)$. Consequently, it is possible to show that $b^{m}(m, \bar{t})<b^{m}\left(m, t_{|\mathcal{S}|-1}\right)$. Indeed, suppose the converse. Then to make the quack indifferent between reporting $m$ at $t_{|\mathcal{S}|-1}$ and $\bar{t}$, we must have $b^{-m}(m, \bar{t})<b^{-m}\left(m, t_{|\mathcal{S}|-1}\right)$. But then

$$
w^{c}\left(\beta^{m}\left(m, t_{|\mathcal{S}|-1}\right)\right)-w^{c}\left(\beta^{-m}\left(m, t_{|\mathcal{S}|-1}\right)\right)<w^{c}\left(\beta^{m}(m, \bar{t})\right)-w^{c}\left(\beta^{-m}(m, \bar{t})\right),
$$

which contradicts the fact that $\Delta w_{\eta}(m, \tau)$ is weakly decreasing in $t$ on $\mathcal{S}$ for $m=\eta$.

Finally, remember that for all $t \in \mathcal{S} \backslash\{\hat{t}\}$ we have

$$
|i(m, t)|=\ln \left(\frac{1+\beta^{m}(m, t)}{1+\beta^{-m}(m, t)}\right)=\ln \left(\frac{1+\beta^{m}(m, t)}{1+\frac{1-\pi}{\pi} \beta^{m}(m, t)}\right)
$$

which is then a decreasing function of $t$ on $\mathcal{S} \backslash\{\bar{t}\}$ as well because $\ln (1+x)-\ln \left(1+\frac{1-\pi}{\pi} x\right)$ is an increasing function of $x$. For the last two points of $\mathcal{S}$ we have

$$
\left|i\left(m, t_{|\mathcal{S}|-1}\right)\right|-|i(m, \bar{t})|=\ln \left(\frac{1+\beta^{m}\left(m, t_{|\mathcal{S}|-1}\right)}{1+\frac{1-\pi}{\pi} \beta^{m}\left(m, t_{|\mathcal{S}|-1}\right)}\right)-\ln \left(\frac{1+\beta^{m}(m, \bar{t})}{1+\frac{1-\pi}{\pi} \beta^{m}(m, \bar{t})+\frac{2 \pi-1}{\pi} \cdot \frac{\left(1-z_{\bar{t}} r_{\varnothing}^{E}(m, \bar{t})\right.}{r^{Q}(m, t)}}\right)>0,
$$

where the last inequality follows from $\beta^{m}\left(m, t_{|\mathcal{S}|-1}\right)>\beta^{m}(m, \bar{t})$ and the fact that $\pi>\frac{1}{2}$. This concludes the proof of Theorem 1 Proposition 9 for general informative equilibria.

We continue by presenting the proof of Proposition 6, which is a special case of Theorem 1/Proposition 9 for delay equilibria.

Proof of Proposition 6. Let $\left\{r_{\eta}^{\gamma}(m, t)\right\}$ constitute a delay equilibrium on $\mathcal{S}$. First, note that if $b_{t_{1}}=b\left(G, t_{1}\right)=b\left(B, t_{1}\right)$ then $b_{t}=b(m, t)=b_{0}$ for $t \in \mathcal{S} \backslash\{t\}$. It further implies that $b^{m}(m, t)$ is constant on $\mathcal{S} \backslash\{\bar{t}\}$. Therefore, $i(m, t)$ is constant on $\mathcal{S} \backslash\{\bar{t}\}$ as well, as suggested by 21], so we get all three statements. If equilibrium is reticent then all these claims are valid for all $t \in \mathcal{S}$.

To show that $b_{t_{1}}=b\left(G, t_{1}\right)=b\left(B, t_{1}\right)$, proceed by contradiction. If there exists $m \in\{G, B\}$ such that $b_{t_{1}}>b\left(m, t_{1}\right)$ then $r_{m}^{E}(m, t)=1$ for all $t \in \mathcal{S} \backslash\left\{t_{|\mathcal{S}|-1}\right\}{ }^{30}$ Due to (SY), the same applies to the other $m$ as well. Further, if such $m$ exists then, as shown above, $b^{m}(m, \bar{t})<b^{m}\left(m, t_{|\mathcal{S}|-1}\right)$, meaning that

$$
w^{c}\left(\beta^{m}\left(m, t_{|\mathcal{S}|-1}\right)\right)-w^{c}\left(\frac{1-\pi}{\pi} \beta^{m}\left(m, t_{|\mathcal{S}|-1}\right)\right)>w^{c}\left(\beta^{m}(m, \bar{t})\right)-w^{c}\left(\frac{1-\pi}{\pi} \beta^{m}(m, \bar{t})\right)
$$

It follows from the fact that $w^{c}\left(x_{1}\right)-w^{c}\left(\frac{1-\pi}{\pi} x_{1}\right)>w^{c}\left(x_{2}\right)-w^{c}\left(\frac{1-\pi}{\pi} x_{2}\right)$ if and only if $x_{1}>x_{2}$ whenever $\pi=1$ (which corresponds to Theorem 1) or any of the two conditions in Proposition 9 are satisfied. Finally, (18) implies that $\frac{1-\pi}{\pi} \beta^{m}\left(m, t_{|\mathcal{S}|-1}\right)=\beta^{-m}\left(m, t_{|\mathcal{S}|-1}\right)$ and $\frac{1-\pi}{\pi} \beta^{m}(m, \bar{t})<\beta^{-m}(m, \bar{t})$, which together give

$$
w^{c}\left(\beta^{m}\left(m, t_{|\mathcal{S}|-1}\right)\right)-w^{c}\left(\beta^{-m}\left(m, t_{|\mathcal{S}|-1}\right)\right)>w^{c}\left(\beta^{m}(m, \bar{t})\right)-w^{c}\left(\beta^{-m}(m, \bar{t})\right) .
$$

The resulting inequality means that informed expert strictly prefers to report his private information at $t_{|\mathcal{S}|-1}$ rather than at $\bar{t}$. This is the last step towards the conclusion that if there exists $m \in\{G, B\}$ such that $b_{t_{1}}>b\left(m, t_{1}\right)$ then $r_{m}^{E}(m, t)=1$ for $m \in\{G, B\}$ and all $t \in \mathcal{S}$, which is a contradiction to $\left\{r_{\eta}^{\gamma}(m, t)\right\}$ constituting a delay equilibrium.

\footnotetext{
${ }^{30}$ The claim for all points except the two last ones follows from the fact that $b^{m}(m, t)$ is strictly decreasing in this case. Furthermore, remember that $r_{m}^{E}(m, \bar{t})=1$ for $m \in\{G, B\}$ by Proposition 2
} 


\section{Proofs for Sections 5.5 and 5.6}

Proving statements about equilibrium existence and properties requires showing some supplementary results first. We start with a lemma that shows that delay equilibria can effectively be considered as modifications of relay equilibria.

Lemma 10. For any delay equilibrium on $\mathcal{S}$ with $|\mathcal{S}| \geqslant 3$ there exists a payoff-equivalent relay equilibrium, such that beliefs after the same histories coincide in the two equilibria.

Proof. Assume that strategy profile $\left\{r_{\eta}^{\gamma}(m, t)\right\}$ constitutes a delay equilibrium on $\mathcal{S}$. Consider strategy profile $\left\{\tilde{r}_{\eta}^{\gamma}(m, t)\right\}$ such that

1. $\tilde{r}_{\eta}^{E}(m, \bar{t})=r_{\eta}^{E}(m, \bar{t})$ and $\tilde{r}^{Q}(m, \bar{t})=r^{Q}(m, \bar{t})$ for $\eta \in\{\varnothing, G, B\}$ and $m \in\{G, B\}$;

2. $\tilde{r}_{\eta}^{E}(m, t)=1$ for $m=\eta, \tilde{r}_{\eta}^{E}(m, t)=0$ for $m \neq \eta$, and $\tilde{r}^{Q}(m, t)=\frac{r^{Q}(m, t)}{r_{m}^{E}(m, t)}$ for all $t \in \mathcal{S} \backslash\{\bar{t}\}$.

By Proposition 6 a strategy profile constitutes a delay equilibrium on $\mathcal{S}$ with $|\mathcal{S}| \geqslant 3$ only if $b_{t}=b(G, t)=$ $b(B, t)=b_{0}$ for all $t \in \mathcal{S} \backslash\{\bar{t}\}$. Therefore $r^{Q}(m, t)=r^{E}(m, t)$ for $m \in\{G, B\}$ and all $t \in \mathcal{S} \backslash\{\bar{t}\}$. Consequently, $\tilde{r}^{Q}(m, t)=\frac{r^{Q}(m, t)}{r_{m}^{E}(m, t)}<\frac{r^{Q}(m, t)}{r^{E}(m, t)}=1$, that is $\tilde{r}_{\eta}^{E}(m, t)=1$ is indeed a well-defined profile of strategies. Moreover, profile $\left\{\tilde{r}_{\eta}^{\gamma}(m, t)\right\}$ induces the same beliefs as profile $\left\{r_{\eta}^{\gamma}(m, t)\right\}$ after the same histories, and therefore also constitutes an equilibrium. At the same time, this equilibrium is a relay one because $r_{G}^{E}(G, m)=r_{B}^{E}(B, m)=1$.

Next we proceed with describing which conditions are necessary for a given profile of strategies $\left\{r_{\eta}^{\gamma}(m, t)\right\}$ to constitute a relay equilibrium. We consider two sub-cases depending on whether not making a report by $\bar{t}$ is on equilibrium path.

Lemma 11. Suppose that beliefs $\beta(m, t)$ and $\beta^{\omega}(m, t)$ for all $t \in \mathcal{S}$ are given by 17) and 18 respectively, while $\beta_{t}$ is given by (19) for all $t<\bar{t}$. Moreover, let strategy profile $\left\{r_{\eta}^{\gamma}(m, t)\right\}$ be such that: (1) $r_{\eta}^{E}(\eta, t)=1$ for all $\eta \in\{G, B\}$ and all $t \in \mathcal{S}$, and (2) $r_{\varnothing}(m, t)=0$ for all $m \in\{G, B\}$ and all $t \in \mathcal{S} \backslash\{t\}$.

1. Strategy profile $\left\{r_{\eta}^{\gamma}(m, t)\right\}$ with $r_{\varnothing}^{E}(G, \bar{t})+r_{\varnothing}^{E}(B, \bar{t})=1$ constitutes a relay equilibrium on $\mathcal{S}$ only if

$$
\begin{aligned}
& W_{t_{1}}^{Q}(m, t)=\bar{W} \text { for all } t \in \mathcal{S} \text { and } m \in\{G, B\} \text { for some } \bar{W} \in \mathbb{R}_{+}, \\
& r^{Q}(G, \bar{t})+r^{Q}(B, \bar{t})=1 .
\end{aligned}
$$

Moreover, there exists at most one solution to this system, and if $w(\beta)=\beta^{\alpha}$ and $w^{c}(\beta)=\theta \cdot \beta^{\alpha}$ then this solution always exists.

2. If (20) holds then strategy profile $\left\{r_{\eta}^{\gamma}(m, t)\right\}$ with $r_{\varnothing}^{E}(G, \bar{t})+r_{\varnothing}^{E}(B, \bar{t})<1$ constitutes a relay equilibrium on $\mathcal{S}$ only if

$$
W_{t_{1}}^{Q}(m, t)=W_{t_{1}}^{Q}(\varnothing)=\bar{W} \text { for all } t \in \mathcal{S} \text { and } m \in\{G, B\} \text { for some } \bar{W} \in \mathbb{R}_{+} .
$$

Moreover, there exists at most one solution to this system, and if $w(\beta)=\beta^{\alpha}$ and $w^{c}(\beta)=\theta \cdot \beta^{\alpha}$ then this solution always exists.

Proof. By Proposition 1, a strategy profile constitutes an equilibrium only if $W_{t_{1}}^{Q}(m, t)$ is constant for all $t \in \mathcal{S}$ and $m \in\{G, B\}$. Additionally, if $r_{\varnothing}^{E}(G, \bar{t})+r_{\varnothing}^{E}(B, \bar{t})<1-$ that is, not making a report by $\bar{t}$ is an on-path action - the value that the quack receives from making any report must be equal to value from making no report. 
The proof of Proposition 1 argued that $r^{E}(G, \bar{t})+r^{E}(B, \bar{t})=1$ implies $r^{Q}(G, \bar{t})+r^{Q}(B, \bar{t})=1$. From Proposition 2 we know that $r_{\eta}^{E}(\eta, \bar{t})=1$, and therefore $r^{E}(G, \bar{t})+r^{E}(B, \bar{t})=1$ is equivalent to $r_{\varnothing}^{E}(G, \bar{t})+r_{\varnothing}^{E}(B, \bar{t})=1$. This completes the proof of the first parts of both statements.

To prove the uniqueness of solutions to respective systems, assume that there exist two different strategy profiles $\left\{r_{\eta}^{\gamma}(m, t)\right\},\left\{\tilde{r}_{\eta}^{\gamma}(m, t)\right\}$, values of making a report $W_{t}^{Q}(m, \tau), \tilde{W}_{t}^{Q}(m, \tau)$, and belief profiles $b, \tilde{b}$ that solve either system $(22)$ or system $(23)$. Then $r^{Q}\left(G, t_{1}\right) \neq \tilde{r}^{Q}\left(G, t_{1}\right)$, as otherwise equilibria coincide. Indeed, strategies $r_{\eta}^{E}(m, t)=\tilde{r}_{\eta}^{E}(m, t)$ for all $t \in \mathcal{S}$. Therefore, if $r^{Q}\left(G, t_{1}\right)=\tilde{r}^{Q}\left(G, t_{1}\right)$ then $b\left(G, t_{1}\right)=\tilde{b}\left(G, t_{1}\right)$ and $b^{\omega}\left(G, t_{1}\right)=\tilde{b}^{\omega}\left(G, t_{1}\right)$, meaning that $W_{t_{1}}^{Q}\left(G, t_{1}\right)=\tilde{W}_{t_{1}}^{Q}\left(G, t_{1}\right)$. By the first two parts of the lemma, the quack's values $W_{t_{1}}^{Q}(m, t)$ should then coincide for all $m$ and $t \in \mathcal{S}$, which implies $r^{Q}(m, t)=\tilde{r}^{Q}(m, t)-$ a contradiction.

Without loss, assume $r^{Q}\left(G, t_{1}\right)>\tilde{r}^{Q}\left(G, t_{1}\right)$. Then since $W_{t_{1}}^{Q}\left(G, t_{1}\right)=W_{t_{1}}^{Q}\left(B, t_{1}\right)$ and $\tilde{W}_{t_{1}}^{Q}\left(G, t_{1}\right)=$ $\tilde{W}_{t_{1}}^{Q}\left(B, t_{1}\right)$, we must have $r^{Q}\left(B, t_{1}\right)>\tilde{r}^{Q}\left(B, t_{1}\right)$ as well. By 19 this implies that $b_{t_{1}}>\tilde{b}_{t_{1}}$. Consequently, $r^{Q}\left(G, t_{2}\right)>\tilde{r}^{Q}\left(G, t_{2}\right)$ and $r^{Q}\left(B, t_{2}\right)>\tilde{r}^{Q}\left(B, t_{2}\right)$ because $W_{t_{1}}^{Q}\left(m, t_{1}\right)=W_{t_{1}}^{Q}\left(m, t_{2}\right)$ and $\tilde{W}_{t_{1}}^{Q}\left(m, t_{1}\right)=$ $\tilde{W}_{t_{1}}^{Q}\left(m, t_{2}\right)$ for $m \in\{G, B\}$. Iterating this logic further, we obtain that $r^{Q}(G, \bar{t})+r^{Q}(B, \bar{t})>\tilde{r}^{Q}(G, \bar{t})+$ $\tilde{r}^{Q}(B, \bar{t})$. In the context of the first part of the lemma (case $r_{\varnothing}^{E}(G, \bar{t})+r_{\varnothing}^{E}(B, \bar{t})=1$ ), it clearly violates $r^{Q}(G, \bar{t})+r^{Q}(B, \bar{t})=\tilde{r}^{Q}(G, \bar{t})+\tilde{r}^{Q}(B, \bar{t})=1$. In the context of the second part, it implies $b_{\bar{t}}>\tilde{b}_{\bar{t}}$, and therefore $W_{t_{1}}^{Q}(\varnothing)>\tilde{W}_{t_{1}}^{Q}(\varnothing)$ because the payoff that the quack receives from staying silent is pointwise lower in the former equilibrium. At the same time, because $r^{Q}\left(G, t_{1}\right)>\tilde{r}^{Q}\left(G, t_{1}\right)$, we must have $W_{t_{1}}^{Q}\left(G, t_{1}\right)<\tilde{W}_{t_{1}}^{Q}\left(G, t_{1}\right)$. As in the second case $W_{t_{1}}^{Q}(\varnothing)=W_{t_{1}}^{Q}\left(G, t_{1}\right)$ and $\tilde{W}_{t_{1}}^{Q}(\varnothing)=\tilde{W}_{t_{1}}^{Q}\left(G, t_{1}\right)$, we arrive to a contradiction.

Finally, to prove existence of a solution for $w(\beta)=\beta^{\alpha}$ and $w^{c}(\beta)=\theta \cdot \beta^{\alpha}$ assume first that $r_{\varnothing}^{E}(G, \bar{t})+r_{\varnothing}^{E}(B, \bar{t})=1$. The first part of the lemma then implies that we have $W_{\bar{t}}^{Q}(G, \bar{t})=W_{\bar{t}}^{Q}(B, \bar{t})$ and $r^{Q}(G, \bar{t})+r^{Q}(B, \bar{t})=1$. We can then explicitly solve this system of equations for $r^{Q}(G, \bar{t})$ and $r^{Q}(B, \bar{t})$ as functions of $r_{\varnothing}^{E}(G, \bar{t})$ and $r_{\varnothing}^{E}(B, \bar{t})$. The resulting expressions are

$$
\begin{aligned}
& r^{Q}(G, \bar{t})=\frac{M_{G}\left(r_{\varnothing}^{E}(G, \bar{t})\right)^{\frac{1}{\alpha}}}{M_{G}\left(r_{\varnothing}^{E}(G, \bar{t})\right)^{\frac{1}{\alpha}}+M_{B}\left(r_{\varnothing}^{E}(B, \bar{t})\right)^{\frac{1}{\alpha}}}, \\
& r^{Q}(B, \bar{t})=\frac{M_{B}\left(r_{\varnothing}^{E}(B, \bar{t})\right)^{\frac{1}{\alpha}}}{M_{G}\left(r_{\varnothing}^{E}(G, \bar{t})\right)^{\frac{1}{\alpha}}+M_{B}\left(r_{\varnothing}^{E}(B, \bar{t})\right)^{\frac{1}{\alpha}}},
\end{aligned}
$$

where

$$
\begin{aligned}
& M_{G}(x):=(T-\bar{t}) \cdot\left(\tilde{p}_{0} z_{\bar{t}}+\left(1-z_{\bar{t}}\right) x\right)^{\alpha}+\theta \cdot p_{0}\left(\pi z_{\bar{t}}+\left(1-z_{\bar{t}}\right) x\right)^{\alpha}+\theta \cdot\left(1-p_{0}\right)\left((1-\pi) z_{\bar{t}}+\left(1-z_{\bar{t}}\right) x\right)^{\alpha}, \\
& M_{B}(x):=(T-\bar{t}) \cdot\left(\left(1-\tilde{p}_{0}\right) z_{\bar{t}}+\left(1-z_{\bar{t}}\right) x\right)^{\alpha}+\theta \cdot p_{0}\left((1-\pi) z_{\bar{t}}+\left(1-z_{\bar{t}}\right) x\right)^{\alpha}+\theta \cdot\left(1-p_{0}\right)\left(\pi z_{\bar{t}}+\left(1-z_{\bar{t}}\right) x\right)^{\alpha} .
\end{aligned}
$$

In case $r_{\varnothing}^{E}(G, \bar{t})+r_{\varnothing}^{E}(B, \bar{t})<1$, the second part of the lemma prescribes that $W_{\bar{t}}^{Q}(G, \bar{t})=W_{\bar{t}}^{Q}(B, \bar{t})=$ $W_{\bar{t}}^{Q}(\varnothing)$. Analogously to the previous case, we can solve for $r^{Q}(G, \bar{t})$ and $r^{Q}(B, \bar{t})$ as functions of $r_{\varnothing}^{E}(G, \bar{t})$ and $r_{\varnothing}^{E}(B, \bar{t})$ and obtain

$$
\begin{aligned}
r^{Q}(G, \bar{t}) & =\frac{M_{G}\left(r_{\varnothing}^{E}(G, \bar{t})\right)^{\frac{1}{\alpha}}}{M_{G}\left(r_{\varnothing}^{E}(G, \bar{t})\right)^{\frac{1}{\alpha}}+M_{B}\left(r_{\varnothing}^{E}(B, \bar{t})\right)^{\frac{1}{\alpha}}+\left(1-z_{\bar{t}}\right) \cdot(T-\bar{t}+\theta)^{\frac{1}{\alpha}} \cdot\left(1-r_{\varnothing}^{E}(G, \bar{t})-r_{\varnothing}^{E}(B, \bar{t})\right)}, \\
r^{Q}(B, \bar{t}) & =\frac{M_{B}\left(r_{\varnothing}^{E}(B, \bar{t})\right)^{\frac{1}{\alpha}}}{M_{G}\left(r_{\varnothing}^{E}(G, \bar{t})\right)^{\frac{1}{\alpha}}+M_{B}\left(r_{\varnothing}^{E}(B, \bar{t})\right)^{\frac{1}{\alpha}}+\left(1-z_{\bar{t}}\right) \cdot(T-\bar{t}+\theta)^{\frac{1}{\alpha}} \cdot\left(1-r_{\varnothing}^{E}(G, \bar{t})-r_{\varnothing}^{E}(B, \bar{t})\right)},
\end{aligned}
$$

Note that expressions in (24) can be obtained from the respective ones in (25) substituting $r_{\varnothing}^{E}(G, \bar{t})+$ $r_{\varnothing}^{E}(B, \bar{t})=1$. Therefore, without loss we can restrict ourselves to the case $r_{\varnothing}^{E}(G, \bar{t})+r_{\varnothing}^{E}(B, \bar{t})<1$ and only consider strategy profile given by (25). All above proves existence of the solution for $t=\bar{t}$. 
We establish existence for all $t \in \mathcal{S} \backslash\{\bar{t}\}$ proceeding by backward induction. Consider system

$$
W_{t_{|\mathcal{S}|-1}}^{Q}\left(G, t_{|\mathcal{S}|-1}\right)=W_{t_{|\mathcal{S}|-1}}^{Q}\left(B, t_{|\mathcal{S}|-1}\right)=W_{t_{|\mathcal{S}|-1}}^{Q}(G, \bar{t})
$$

We next show that this system of equations always has a solution. Consider the following auxiliary system. For any given $c>0$ assume that $r^{Q}\left(G, t_{|\mathcal{S}|-1}\right)+r^{Q}\left(B, t_{|\mathcal{S}|-1}\right)=c$ and consider equation

$$
W_{t_{|\mathcal{S}|-1}}^{Q}\left(G, t_{|\mathcal{S}|-1}\right)=W_{t_{|\mathcal{S}|-1}}^{Q}\left(B, t_{|\mathcal{S}|-1}\right)
$$

Then if $r^{Q}\left(G, t_{|\mathcal{S}|-1}\right)$ approaches zero, the LHS approaches $+\infty$ while the RHS is constant. Similarly, the RHS strictly dominates the LHS when $r^{Q}\left(G, t_{|\mathcal{S}|-1}\right)=c$. Moreover the LHS is strictly decreasing in $r^{Q}\left(G, t_{|\mathcal{S}|-1}\right)$, while the RHS is strictly increasing in it. Therefore by the Intermediate Value Theorem for a given $c>0$ there exists a unique pair $r^{Q}\left(G, t_{|\mathcal{S}|-1}\right), r^{Q}\left(B, t_{|\mathcal{S}|-1}\right) \operatorname{such}$ that $r^{Q}\left(G, t_{|\mathcal{S}|-1}\right)+r^{Q}\left(B, t_{|\mathcal{S}|-1}\right)=c$ and $W_{t_{|\mathcal{S}|-1}}^{Q}\left(G, t_{|\mathcal{S}|-1}\right)=W_{t_{|\mathcal{S}|-1}}^{Q}\left(B, t_{|\mathcal{S}|-1}\right)$. Also note that both $r^{Q}\left(G, t_{|\mathcal{S}|-1}\right)$ and $r^{Q}\left(B, t_{|\mathcal{S}|-1}\right)$ are strictly increasing in $c$. Further for the same $c>0$ still assume that $r^{Q}\left(G, t_{|\mathcal{S}|-1}\right)+r^{Q}\left(B, t_{|\mathcal{S}|-1}\right)=c$ and consider equality

$$
W_{t_{|\mathcal{S}|-1}}^{Q}\left(G, t_{|\mathcal{S}|-1}\right)=W_{t_{|\mathcal{S}|-1}}^{Q}(G, \bar{t})
$$

as an equation in $c$. The RHS of it is a strictly increasing function of $c$ which approaches $+\infty$ when $c$ approaches 1. As established before, the LHS of it is a strictly decreasing function of $c$ (because $r^{Q}\left(G, t_{|\mathcal{S}|-1}\right)$ is strictly increasing in $c$ ), which approaches $+\infty$ when $c$ approaches zero. Therefore there exist unique $r^{Q}\left(G, t_{|\mathcal{S}|-1}\right)$ and $r^{Q}\left(B, t_{|\mathcal{S}|-1}\right)$ such that $W_{t_{|\mathcal{S}|-1}}^{Q}\left(G, t_{|\mathcal{S}|-1}\right)=W_{t_{|\mathcal{S}|-1}}^{Q}\left(B, t_{|\mathcal{S}|-1}\right)=W_{t_{|\mathcal{S}|-1}}^{Q}(G, \bar{t})$, which finishes the proof.

The bottom line of the lemma above is that for a given tuple $\left[\mathcal{S}, r_{\varnothing}^{E}(G, \bar{t}), r_{\varnothing}^{E}(B, \bar{t})\right]$, a strategy profile that constitutes a relay equilibrium is a unique solution to a particular system of algebraic equations. Moreover, solution to this system always exists if $w(\beta)=\beta^{\alpha}$ and $w^{c}(\beta)=\theta \cdot \beta^{\alpha}$. Representing a strategy profile as a solution to a system of equations allows us to compare equilibrium strategies and, therefore, report informativeness across different relay equilibria employing the arguments similar to the Implicit Function Theorem.

In all further lemmas it is assumed that strategy profile $r_{\eta}^{\gamma}(m, t)$ and all associated equilibrium objects such as values $W_{t}^{Q}(m, \tau)$, belief profiles $b, p$, and informativeness measures $i(m, t)$ constitute a solution to either system 22 or system 23 for a given tuple $\left[\mathcal{S}, r_{\varnothing}^{E}(G, \bar{t}), r_{\varnothing}^{E}(B, \bar{t})\right]$, and therefore are understood as functions of $\left[\mathcal{S}, r_{\varnothing}^{E}(G, \bar{t}), r_{\varnothing}^{E}(B, \bar{t})\right]$.

The next lemma establishes that whenever $w(\beta)=\beta^{\alpha}$ and $w^{c}(\beta)=\theta \cdot \beta^{\alpha}$, it is true that strategies that constitute a solution to either system 22 or system 23 are continuously differentiable in $r_{\varnothing}^{E}(G, \bar{t})$ and $r_{\varnothing}^{E}(B, \bar{t})$ at $r_{\varnothing}^{E}(G, \bar{t})+r_{\varnothing}^{E}(B, \bar{t})=1$. The same will then be true of all associated equilibrium objects $W_{t}^{Q}(m, \tau), b$ and $i(m, t)$, as they all are continuously differentiable functions of the strategies. The statement of this lemma is valid for any continuously differentiable $w(\cdot)$, but the statement for this particular functional form is enough for the needs of the paper and is significantly easier to prove. Lemma 12 allows us to further omit the consideration of the case $r_{\varnothing}^{E}(G, \bar{t})+r_{\varnothing}^{E}(B, \bar{t})=1$ and without loss assume in further propositions that $r_{\varnothing}^{E}(G, \bar{t})+r_{\varnothing}^{E}(B, \bar{t})<1$.

Lemma 12. Suppose $w(\beta)=\beta^{\alpha}$ and $w^{c}(\beta)=\theta \cdot \beta^{\alpha}$, and strategy profile $\left\{r_{\eta}^{\gamma}(m, t)\right\}$ solves either system 22 or system (23). Then $r_{\eta}^{\gamma}(m, t)$ is a continuously differentiable function of $r_{\varnothing}^{E}(G, \bar{t})$ and $r_{\varnothing}^{E}(B, \bar{t})$ for all $r_{\varnothing}^{E}(G, \bar{t})+r_{\varnothing}^{E}(B, \bar{t}) \leqslant 1$. 
Proof. First note that $r_{\eta}^{\gamma}(m, t)$ exists by Lemma 11. Next, the strategy profile for the expert is fixed by the premise of Lemma 11 and is therefore a continuously differentiable function of $r_{\varnothing}^{E}(G, \bar{t})$ and $r_{\varnothing}^{E}(B, \bar{t})$. Therefore we are left to establish that $r^{Q}(m, t)$ is a continuously differentiable function of $r_{\varnothing}^{E}(G, \bar{t})$ and $r_{\varnothing}^{E}(B, \bar{t})$ for all $m \in\{G, B\}$ and all $t \in \mathcal{S}$. As expressions in (24) coincide with the ones in (25) for $r_{\varnothing}^{E}(G, \bar{t})+r_{\varnothing}^{E}(B, \bar{t})=1$, without loss, we restrict ourselves to the case $r_{\varnothing}^{E}(G, \bar{t})+r_{\varnothing}^{E}(B, \bar{t})<1$. Both expressions in 25) are continuously differentiable functions of $r_{\varnothing}^{E}(G, \bar{t})$ and $r_{\varnothing}^{E}(B, \bar{t})$ for $r_{\varnothing}^{E}(G, \bar{t})+r_{\varnothing}^{E}(B, \bar{t}) \leqslant$ 1. Thus, it is left to show the same for $r^{Q}(G, t)$ and $r^{Q}(B, t)$ for $t \in \mathcal{S} \backslash\{\bar{t}\}$. We proceed using backward induction. Consider two equalities

$$
\begin{aligned}
& W_{t_{|\mathcal{S}|-1}}^{Q}\left(G, t_{|\mathcal{S}|-1}\right)=W_{t_{|\mathcal{S}|-1}}^{Q}(G, \bar{t}) \\
& W_{t_{|\mathcal{S}|-1}}^{Q}\left(B, t_{|\mathcal{S}|-1}\right)=W_{t_{|\mathcal{S}|-1}}^{Q}(B, \bar{t}) .
\end{aligned}
$$

Given $r^{Q}(G, \bar{t})$ and $r^{Q}(B, \bar{t})$, they constitute a system of equations on $r^{Q}\left(G, t_{|\mathcal{S}|-1}\right)$ and $r^{Q}\left(B, t_{|\mathcal{S}|-1}\right)$. Moreover, because $w(\beta)=\beta^{\alpha}$, both $r^{Q}\left(G, t_{|\mathcal{S}|-1}\right)$ and $r^{Q}\left(B, t_{|\mathcal{S}|-1}\right)$ do not depend on $b_{t_{|\mathcal{S}|-2}}$ that is, on strategies $r^{Q}(G, t)$ and $r^{Q}(B, t)$ for $t \leqslant t_{|\mathcal{S}|-2}-$ but only on $r^{Q}(G, \bar{t})$ and $r^{Q}(B, \bar{t})$. Therefore, by the Implicit Function Theorem, $r^{Q}\left(G, t_{|\mathcal{S}|-1}\right)$ and $r^{Q}\left(B, t_{|\mathcal{S}|-1}\right)$ are continuously differentiable functions of $r^{Q}(G, \bar{t})$ and $r^{Q}(B, \bar{t})$, which eventually implies that they are continuously differentiable functions of $r_{\varnothing}^{E}(G, \bar{t})$ and $r_{\varnothing}^{E}(B, \bar{t})$. Proceeding backwards we establish the claim for all $r^{Q}(G, t)$ and $r^{Q}(B, t)$ for $t \in \mathcal{S}$.

The two following lemmas are mostly technical and provide little intuition for the main problem.

Lemma 13. Suppose

$$
m(x)=\left(\chi_{1}\left(a_{1}+b x\right)^{\alpha}+\ldots+\chi_{k}\left(a_{k}+b x\right)^{\alpha}\right)^{\frac{1}{\alpha}}-b x
$$

for $k \geqslant 2, b>0, \sum_{i=1}^{k} \chi_{i}=1, a_{1}, \ldots, a_{k} \geqslant 0$ with $a_{i}, a_{j}>0$ for some $i, j \in\{1, \ldots, k\}, i \neq j$. Then $m(x) i s$ strictly decreasing when $\alpha>1$ and is strictly increasing when $\alpha<1$.

Proof. Begin by observing that

$$
\frac{1}{b} \cdot \frac{d m(x)}{d x}=\frac{\chi_{1}\left(a_{1}+b x\right)^{\alpha-1}+\ldots+\chi_{k}\left(a_{k}+b x\right)^{\alpha-1}}{\left(\chi_{1}\left(a_{1}+b x\right)^{\alpha}+\ldots+\chi_{k}\left(a_{k}+b x\right)^{\alpha}\right)^{\frac{\alpha-1}{\alpha}}}-1
$$

First, if $\alpha>1$ then, since $x^{k}$ is strictly convex for $k>1$, we have

$$
\left(\chi_{1}\left(a_{1}+b x\right)^{\alpha-1}+\ldots+\chi_{k}\left(a_{k}+b x\right)^{\alpha-1}\right)^{\frac{\alpha}{\alpha-1}}<\chi_{1}\left(a_{1}+b x\right)^{\alpha}+\ldots+\chi_{k}\left(a_{k}+b x\right)^{\alpha}
$$

and therefore $\frac{d m(x)}{d x}<0$ if $\alpha>1$.

Second, if $\alpha<1$ then, because $x^{k}$ is strictly convex for $k<0$, we still have 26 satisfied. Therefore, because $\frac{\alpha-1}{\alpha}<0$, we have

$$
\chi_{1}\left(a_{1}+b x\right)^{\alpha-1}+\ldots+\chi_{k}\left(a_{k}+b x\right)^{\alpha-1}>\left(\chi_{1}\left(a_{1}+b x\right)^{\alpha}+\ldots+\chi_{k}\left(a_{k}+b x\right)^{\alpha}\right)^{\frac{\alpha-1}{\alpha}},
$$

and therefore $\frac{d m(x)}{d x}>0$ if $\alpha<1$.

Lemma 14. Suppose $w(\beta)=\beta^{\alpha}$, and strategy profile $\left\{r_{\eta}^{\gamma}(m, t)\right\}$ solves either system (22) or system 23. 
Then if $\alpha<1$ for any $m \in\{G, B\}$ we have

$$
\frac{\partial}{\partial r_{\varnothing}^{E}(m, \bar{t})}\left(\frac{\left(1-z_{\bar{t}}\right) \cdot\left(1-r_{\varnothing}^{E}(G, \bar{t})-r_{\varnothing}^{E}(B, \bar{t})\right)}{1-r^{Q}(G, \bar{t})-r^{Q}(B, \bar{t})}\right)>0,
$$

while if $\alpha>1$ for any $m \in\{G, B\}$ we have

$$
\frac{\partial}{\partial r_{\varnothing}^{E}(m, \bar{t})}\left(\frac{\left(1-z_{\bar{t}}\right) \cdot\left(1-r_{\varnothing}^{E}(G, \bar{t})-r_{\varnothing}^{E}(B, \bar{t})\right)}{1-r^{Q}(G, \bar{t})-r^{Q}(B, \bar{t})}\right)<0 .
$$

Additionally, for any $m \in\{G, B\}$ and any $t \in \mathcal{S}$ we have that $W_{t_{1}}^{Q}(m, t)$ is strictly increasing in $r_{\varnothing}^{E}(m, \bar{t})$ if $\alpha<1$, and $W_{t_{1}}^{Q}(m, t)$ is strictly decreasing in $r_{\varnothing}^{E}(m, \bar{t})$ if $\alpha<1$.

Proof. Lemmas 11 and 12 imply that $r^{Q}(m, t)$ exists for all $m \in\{G, B\}$ and $t \in \mathcal{S}$ and is continuously differentiable in $r_{\varnothing}^{E}(G, \bar{t})$ and $r_{\varnothing}^{E}(B, \bar{t})$. Next, from $(25)$ we can calculate that

$$
\frac{\left(1-z_{\bar{t}}\right) \cdot\left(1-r_{\varnothing}^{E}(G, \bar{t})-r_{\varnothing}^{E}(G, \bar{t})\right)}{1-r^{Q}(G, \bar{t})-r^{Q}(B, \bar{t})}=\frac{M_{G}\left(r_{\varnothing}^{E}(G, \bar{t})\right)^{\frac{1}{\alpha}}+M_{B}\left(r_{\varnothing}^{E}(B, \bar{t})\right)^{\frac{1}{\alpha}}}{(T-\bar{t}+\theta)^{\frac{1}{\alpha}}}+\left(1-z_{\bar{t}}\right) \cdot\left(1-r_{\varnothing}^{E}(G, \bar{t})-r_{\varnothing}^{E}(B, \bar{t})\right),
$$

which is the sum of two functions of the form from Lemma 13 and a constant. Therefore, the first statement of the Lemma follows directly from Lemma 13.

Next we establish the second claim. By Lemma 12 we can assume without loss that $r_{\varnothing}^{E}(G, \bar{t})+r_{\varnothing}^{E}(B, \bar{t})<$ 1. What follows is the proof for the case $\alpha<1$ (case $\alpha>1$ is analogous). For a given $m \in\{G, B\}$ fix some $r_{\varnothing}^{E}(m, \bar{t})<\tilde{r}_{\varnothing}^{E}(m, \bar{t})$ and $r_{\varnothing}^{E}(-m, \bar{t})=\tilde{r}_{\varnothing}^{E}(-m, \bar{t})$. Also denote the respective strategy profiles that solve system $\left[23\right.$ for $\left[\mathcal{S}, r_{\varnothing}^{E}(G, \bar{t}), r_{\varnothing}^{E}(B, \bar{t})\right]$ and $\left[\mathcal{S}, \tilde{r}_{\varnothing}^{E}(G, \bar{t}), \tilde{r}_{\varnothing}^{E}(B, \bar{t})\right]$ as $\left\{r_{\eta}^{\gamma}(m, t)\right\}$ and $\left\{\tilde{r}_{\eta}^{\gamma}(m, t)\right\}$. Denote by $b$ and $\tilde{b}$ the respective beliefs, and by $W_{t}^{\gamma}(m, \tau)$ and $\tilde{W}_{t}^{\gamma}(m, \tau)$ the respective values from reports.

Assume that $W_{t_{1}}^{Q}(m, t) \geqslant \tilde{W}_{t_{1}}^{Q}(m, t)$. Then $r^{Q}\left(m, t_{1}\right) \leqslant \tilde{r}^{Q}\left(m, t_{1}\right)$ for $m \in\{G, B\}$. From 19) we then get that $b_{t_{1}} \leqslant \tilde{b}_{t_{1}}$. This, in turn, implies that $r^{Q}\left(G, t_{2}\right) \leqslant \tilde{r}^{Q}\left(G, t_{2}\right)$ and $r^{Q}\left(B, t_{2}\right) \leqslant \tilde{r}^{Q}\left(B, t_{2}\right)$ because $W_{t_{1}}^{Q}\left(m, t_{1}\right)=W_{t_{1}}^{Q}\left(m, t_{2}\right)$ and $\tilde{W}_{t_{1}}^{Q}\left(m, t_{1}\right)=\tilde{W}_{t_{1}}^{Q}\left(m, t_{2}\right)$ for $m \in\{G, B\}$. Iterating this logic further, we get $b_{t_{|\mathcal{S}-1|}} \leqslant \tilde{b}_{t_{|\mathcal{S}-1|}}$. From the first part of the lemma we know that

$$
\frac{\left(1-z_{\bar{t}}\right) \cdot\left(1-\tilde{r}_{\varnothing}^{E}(G, \bar{t})-\tilde{r}_{\varnothing}^{E}(G, \bar{t})\right)}{1-\tilde{r}^{Q}(G, \bar{t})-\tilde{r}^{Q}(B, \bar{t})}>\frac{\left(1-z_{\bar{t}}\right) \cdot\left(1-r_{\varnothing}^{E}(G, \bar{t})-r_{\varnothing}^{E}(G, \bar{t})\right)}{1-r^{Q}(G, \bar{t})-r^{Q}(B, \bar{t})}
$$

and therefore $W_{t_{1}}^{Q}(\varnothing)<\tilde{W}_{t_{1}}^{Q}(\varnothing)$. This gives us a contradiction with the initial assumption $W_{t_{1}}^{Q}(m, t) \geqslant$ $\tilde{W}_{t_{1}}^{Q}(m, t)$ because we must have $W_{t_{1}}^{Q}(\varnothing)=W_{t_{1}}^{Q}\left(G, t_{1}\right)$ and $\tilde{W}_{t_{1}}^{Q}(\varnothing)=\tilde{W}_{t_{1}}^{Q}\left(G, t_{1}\right)$.

Proof of Proposition 4. Part 1. We first show that for any set of parameters $|\mathcal{S}|=1, r_{\varnothing}^{E}(G, \bar{t})>0$, and $r_{\varnothing}^{E}(B, \bar{t})>0$, the informative equilibrium with given parameters exists. Proposition 2 and the values of $r_{\varnothing}^{E}(m, t)$ pin down the expert's strategy. We next show that there exists such a quack's strategy $r^{Q}(m, \bar{t})$ that conditions in Lemma 11 are satisfied, which proves this part of the proposition. Note also that for singleton $\mathcal{S}$ we have $z_{\bar{t}}=F(t)$.

The first condition one needs to check in order to establish existence is $W_{\bar{t}}^{Q}(G, \bar{t})=W_{\bar{t}}^{Q}(B, \bar{t})$, which can be written as

$$
\begin{aligned}
& (T-\bar{t}) \cdot w(\beta(G, \bar{t}))+p_{0} w^{c}\left(\beta^{G}(G, \bar{t})\right)+\left(1-p_{0}\right) w^{c}\left(\beta^{B}(G, \bar{t})\right)= \\
= & (T-\bar{t}) \cdot w(\beta(B, \bar{t}))+p_{0} w^{c}\left(\beta^{G}(B, \bar{t})\right)+\left(1-p_{0}\right) w^{c}\left(\beta^{B}(B, \bar{t})\right) .
\end{aligned}
$$

From (17) and (18) we see that the LHS is strictly decreasing in $r^{Q}(G, \bar{t})$, and the RHS is strictly increasing 
in $r^{Q}(G, \bar{t})$. Moreover, all six terms in (27) are always positive irrespectively of $r^{Q}(G, \bar{t})$ because $r_{\varnothing}^{E}(G, \bar{t})>0$ and $r_{\varnothing}^{E}(B, \bar{t})>0$. Therefore when $r^{Q}(G, \bar{t})=0$ the LHS strictly dominates the RHS, and when $r^{Q}(G, \bar{t})=1$ the RHS strictly dominates the LHS. By the Intermediate Value Theorem, there exists unique $r^{Q}(G, \bar{t}$ ) (and therefore $\left.r^{Q}(B, \bar{t})\right)$ such that 27 is satisfied.

If $r_{\varnothing}^{E}(G, \bar{t})+r_{\varnothing}^{E}(B, \bar{t})=1$ then we are done. Strictly speaking, this is enough to prove the statement. However, for sake of completeness we show that the equilibrium exists in case $r_{\varnothing}^{E}(G, \bar{t})+r_{\varnothing}^{E}(B, \bar{t})<1$ as well. To do this we need to ensure that $W_{\bar{t}}^{Q}(G, \bar{t})=W_{\bar{t}}^{Q}(\varnothing)$, i.e., that the value of not making a report at $\bar{t}$ is equal to the value of making a report:

$$
(T-\bar{t}) \cdot w(\beta(G, \bar{t}))+p_{0} w^{c}\left(\beta^{G}(G, \bar{t})\right)+\left(1-p_{0}\right) w^{c}\left(\beta^{B}(G, \bar{t})\right)=(T-\bar{t}) \cdot w\left(\beta_{\bar{t}}\right)+w^{c}\left(\beta_{\bar{t}}\right) .
$$

By the same logic as above, we know that for any given $c>0$ there exist unique $r^{Q}(G, \bar{t})$ and $r^{Q}(B, \bar{t})$ such that 27) is satisfied and $r^{Q}(G, \bar{t})+r^{Q}(B, \bar{t})=c$. Further, note that $r_{\varnothing}^{E}(G, \bar{t})$ and $r_{\varnothing}^{E}(B, \bar{t})$ that are obtained as a solution to this auxiliary system are both increasing in $c{ }^{31}$ Finally, consider (28) as an equation in $c$. From the previous observation it follows that its LHS is decreasing in $c$, while the RHS is increasing in $c$. If $c=0$ then the LHS dominates the RHS, and if $c=1$ the RHS dominates the LHS. Therefore, by the Intermediate Value Theorem there exists unique $c$ such that 28) is satisfied. Solving 27) using this $c$ gives a pair $r^{Q}(G, \bar{t})$ and $r^{Q}(B, \bar{t})$ that uniquely solves the original system of 27) and 28.

Part 2. To prove the second part of the proposition we construct a relay reticent equilibrium for a given $\mathcal{S}$ with $|\mathcal{S}| \geqslant 2$ and $r_{\varnothing}^{E}(G, \bar{t})=r_{\varnothing}^{E}(B, \bar{t})=0$. Since states are symmetric, for any $t \in \mathcal{S}$ the quack is indifferent between reports $(G, t)$ and $(B, t)$ if and only if $r^{Q}(G, t)=r^{Q}(B, t)$. Thus, we are only left to ensure the indifference between a report and no report for the quack and to verify that the constructed equilibrium is incentive compatible for informed expert. Define $g:=g\left(G, t_{1}\right)=g\left(B, t_{1}\right)$. Then $W_{t_{1}}^{Q}\left(G, t_{1}\right)=W_{t_{1}}^{Q}\left(B, t_{1}\right)$ is equal to

$$
\left(T-t_{1}\right) \cdot w\left(\beta_{0} \cdot e^{g}\right)+p_{0} w^{c}\left(\beta_{0} \cdot \frac{\pi}{\tilde{p}_{0}} e^{g}\right)+\left(1-p_{0}\right) w^{c}\left(\beta_{0} \cdot \frac{(1-\pi)}{\tilde{p}_{0}} e^{g}\right) .
$$

From the expression above we see that the value of $g$ fully determines the value that the quack gets in equilibrium. In a relay equilibrium the expert's strategy is fixed, so smaller $g$ means $\operatorname{larger} r^{Q}\left(G, t_{1}\right)$ and $r^{Q}\left(B, t_{1}\right)$. Larger $r^{Q}\left(G, t_{1}\right)$ and $r^{Q}\left(B, t_{1}\right)$, in turn, imply higher $b_{t_{1}}$. Finally, because the quack must be indifferent between reports at $t_{1}$ and $t_{2}$, higher $b_{t_{1}}$ implies larger $r^{Q}\left(G, t_{2}\right)$ and $r^{Q}\left(B, t_{2}\right)$. All in all, it means that the payoff that the quack receives by not making a report is point-wise strictly decreasing in $g$. When $g=0$ we have that $b_{t}=b_{0}$ for all $t \in \mathcal{T}$ (remember that $r_{\varnothing}^{E}(G, \bar{t})=r_{\varnothing}^{E}(B, \bar{t})=0$, so following the logic from Proposition 9 we have $g(m, t)=0$ for all $t \in \mathcal{T})$, therefore value of making no report by the end of period $T$ evaluated at $t_{1}$ is equal to $\left(T-t_{1}\right) \cdot w\left(\beta_{0}\right)+w^{c}\left(\beta_{0}\right)$. When $g \rightarrow-\infty$ we have that the value of making no report strictly dominates the value of making a report. When $g=0$ we have

$$
\left(T-t_{1}\right) \cdot w\left(\beta_{0}\right)+p_{0} w^{c}\left(\beta_{0} \cdot \frac{\pi}{\tilde{p}_{0}}\right)+\left(1-p_{0}\right) w^{c}\left(\beta_{0} \cdot \frac{(1-\pi)}{\tilde{p}_{0}}\right) \geqslant\left(T-t_{1}\right) \cdot w\left(\beta_{0}\right)+w^{c}\left(\beta_{0}\right)
$$

because $w^{c}(\cdot)$ is convex. Therefore, by the Intermediate Value Theorem, there exists a unique $g \leqslant 0$ such that the quack is indifferent between making a report and not making a report. Finally, because $g\left(G, t_{1}\right)=g\left(B, t_{1}\right)=g \leqslant 0$ we have $b\left(m, t_{1}\right) \leqslant b_{0} \leqslant b_{t_{1}}$. From Proposition 9 for convex $w^{c}(\cdot)$ we know that it implies that $b^{m}(m, t)$ - and, consequently, $\Delta w_{\eta}(m, t)$ for $m=\eta$ - are decreasing on $\mathcal{S}$, which verifies that $r_{\eta}^{E}(\eta, t)=1$ is an optimal strategy for the expert, i.e., the constructed profile indeed constitutes an

\footnotetext{
${ }^{31}$ At least one of $r_{\varnothing}^{E}(G, \bar{t})$ and $r_{\varnothing}^{E}(B, \bar{t})$ must be higher for a higher $c$, and 27$]$ implies that higher $r_{\varnothing}^{E}(G, \bar{t})$ implies higher $r_{\varnothing}^{E}(B, \bar{t})$.
} 
equilibrium.

Part 3. To prove the third part assume the contrary: there exists $\mathcal{S}$ with $|\mathcal{S}| \geqslant 3$ such that informative equilibrium with respective strategy profile $\left\{r_{\eta}^{\gamma}(m, t)\right\}$ for $t \in \mathcal{S}$ exists. By Lemma 10 we can assume without loss that the equilibrium is a relay one. By Lemma 11 we know that there exists a strategy profile $\left\{\tilde{r}_{\eta}^{\gamma}(m, t)\right\}$ (and associated belief profile $\tilde{b}$ and value function $\tilde{W}_{t}^{\gamma}(m, \tau)$ ) for the same $\mathcal{S}$ with $\tilde{r}_{\varnothing}^{E}(G, t)=\tilde{r}_{\varnothing}^{E}(B, t)=0$ which solves system 23 . We next show that this profile constitutes a relay equilibrium on $\mathcal{S}$. The only condition that needs to be verified is that this profile is incentive compatible for informed expert. By the proof of Proposition 9 for $\mathcal{S}$ with $|\mathcal{S}| \geqslant 3$ this is equivalent to verifying that $\tilde{b}_{t_{1}} \geqslant \tilde{b}\left(m, t_{1}\right)$ for $m \in\{G, B\}$ because $\tilde{r}_{\varnothing}^{E}(G, t)=\tilde{r}_{\varnothing}^{E}(B, t)=0$.

In the original equilibrium we have $b_{t_{1}} \geqslant b\left(m, t_{1}\right)$ by the same Proposition 9 By Lemma 14 because $r_{\varnothing}^{E}(m, t) \geqslant \tilde{r}_{\varnothing}^{E}(m, t)$ for $m \in\{G, B\}$ and $\alpha<1$, we have $W_{t_{1}}^{Q}\left(m, t_{1}\right)>\tilde{W}_{t_{1}}^{Q}\left(m, t_{1}\right)$. This implies that $r^{Q}\left(m, t_{1}\right)<\tilde{r}^{Q}\left(m, t_{1}\right)$ for $m \in\{G, B\}$, and therefore $\tilde{b}_{t_{1}}>b_{t_{1}} \geqslant b\left(m, t_{1}\right)>\tilde{b}\left(m, t_{1}\right)$ for $m \in\{G, B\}$, which completes the argument.

We have established the existence of the relay equilibrium on $\mathcal{S}$ with $\tilde{r}_{\varnothing}^{E}(G, t)=\tilde{r}_{\varnothing}^{E}(B, t)=0$. By Proposition 9 there exists $m \in\{G, B\}$ such that $\tilde{b}\left(m, t_{1}\right) \leqslant b_{0}$, and therefore

$$
\tilde{W}_{t_{1}}^{Q}\left(m, t_{1}\right) \leqslant\left(T-t_{1}\right) \cdot w\left(\beta_{0}\right)+p_{0} w^{c}\left(\beta_{0} \cdot \frac{\pi}{\tilde{p}_{0}}\right)+\left(1-p_{0}\right) w^{c}\left(\beta_{0} \cdot \frac{1-\pi}{\tilde{p}_{0}}\right) .
$$

At the same time, because in such equilibrium $b_{t} \geqslant b_{0}$ for all $t \in \mathcal{S}$ (again by Proposition 9), we have

$$
\tilde{W}_{t_{1}}^{Q}(\varnothing) \geqslant\left(T-t_{1}\right) \cdot w\left(\beta_{0}\right)+w^{c}\left(\beta_{0}\right)
$$

Finally, $\tilde{W}_{t_{1}}^{Q}\left(m, t_{1}\right)=\tilde{W}_{t_{1}}^{Q}(\varnothing)$ implies

$$
p_{0} w^{c}\left(\beta_{0} \cdot \frac{\pi}{\tilde{p}_{0}}\right)+\left(1-p_{0}\right) w^{c}\left(\beta_{0} \cdot \frac{1-\pi}{\tilde{p}_{0}}\right) \geqslant w^{c}\left(\beta_{0}\right) .
$$

If $w^{c}(\cdot)$ is strictly concave then (29) can not be satisfied, which gives us the contradiction.

Proof of Proposition 5 . Denote by $W_{t}^{\gamma}(m, \tau)$ and $\tilde{W}_{t}^{\gamma}(m, \tau)$ the respective values of making report and by $b$ and $\tilde{b}$ the beliefs for strategy profiles $\left\{r_{\eta}^{\gamma}(m, t)\right\}$ and $\left\{\tilde{r}_{\eta}^{\gamma}(m, t)\right\}$.

To prove the first part of the proposition, we first show that $W_{t_{1}}^{Q}\left(m, t_{1}\right) \leqslant \tilde{W}_{t_{1}}^{Q}\left(m, t_{1}\right)$ for $m \in\{G, B\}$. Assume the contrary, i.e., that $W_{t_{1}}^{Q}\left(m, t_{1}\right)>\tilde{W}_{t_{1}}^{Q}\left(m, t_{1}\right)$ for $\left.m \in\{G, B\}\right\}^{32}$ Then it directly implies $r^{Q}\left(m, t_{1}\right)<\tilde{r}^{Q}\left(m, t_{1}\right)$ for $m \in\{G, B\}$. From 19 it then follows that $b_{t_{1}}<\tilde{b}_{t_{1}}$. This, in turn, implies that $r^{Q}\left(G, t_{2}\right)<\tilde{r}^{Q}\left(G, t_{2}\right)$ and $r^{Q}\left(B, t_{2}\right)<\tilde{r}^{Q}\left(B, t_{2}\right)$ because $W_{t_{1}}^{Q}\left(m, t_{1}\right)=W_{t_{1}}^{Q}\left(m, t_{2}\right)$ and $\tilde{W}_{t_{1}}^{Q}\left(m, t_{1}\right)=\tilde{W}_{t_{1}}^{Q}\left(m, t_{2}\right)$ for $m \in\{G, B\}$. Iterating this logic further, we obtain that $b_{t}<\tilde{b}_{t}$ for all $t \in \mathcal{S}$. Additionally, by Proposition 9 we have $b_{t_{k}} \leqslant b_{t_{k+1}} \leqslant \ldots \leqslant b_{t_{k+n}}$ (we can extend the argument to $b_{t_{k+n}}$ because $\tilde{r}_{\varnothing}^{E}\left(m, t_{n+k}\right)=0$ for $\left.m \in\{G, B\}\right)$. Therefore, $W_{t_{1}}^{Q}(\varnothing)<\tilde{W}_{t_{1}}^{Q}(\varnothing)$. Making no report is an on-path action in both equilibria, thus $W_{t_{1}}^{Q}\left(G, t_{1}\right)=W_{t_{1}}^{Q}(\varnothing)$ and $\tilde{W}_{t_{1}}^{Q}\left(G, t_{1}\right)=\tilde{W}_{t_{1}}^{Q}(\varnothing)$. Consequently, $W_{t_{1}}^{Q}\left(m, t_{1}\right)<\tilde{W}_{t_{1}}^{Q}\left(m, t_{1}\right)$, which gives us a contradiction with the initial assumption.

Condition $W_{t_{1}}^{Q}(m, t) \leqslant \tilde{W}_{t_{1}}^{Q}(m, t)$ directly implies that $r^{Q}\left(m, t_{1}\right) \geqslant \tilde{r}^{Q}\left(m, t_{1}\right)$ - since in a relay equilibrium the strategy of the expert is fixed, - and therefore $|i(m, t)| \leqslant|\tilde{i}(m, t)|$ for all $t \in \mathcal{S}$. Finally, $|i(m, t)|=0$ for any $t \notin \mathcal{S}$, meaning that $|i(m, t)|<\tilde{i}(m, t) \mid$ for $t \in \tilde{\mathcal{S}} \backslash \mathcal{S}$.

\footnotetext{
${ }^{32}$ Because $W_{t_{1}}^{Q}\left(G, t_{1}\right)=W_{t_{1}}^{Q}\left(B, t_{1}\right)$ and $\tilde{W}_{t_{1}}^{Q}\left(G, t_{1}\right)=\tilde{W}_{t_{1}}^{Q}\left(B, t_{1}\right)$, we have that either $W_{t_{1}}^{Q}\left(m, t_{1}\right)>\tilde{W}_{t_{1}}^{Q}\left(m, t_{1}\right)$ for both $m \in\{G, B\}$ or $W_{t_{1}}^{Q}\left(m, t_{1}\right)<\tilde{W}_{t_{1}}^{Q}\left(m, t_{1}\right)$ for both $m \in\{G, B\}$.
} 


\section{Proofs for Section 6}

Proof of Proposition 8. In an ideal equilibrium, $r_{\eta}^{E}(\eta, t)=1$ and $r_{\varnothing}^{E}(m, t)=r^{Q}(m, t)=0$ for $\eta, m \in\{G, B\}$ and all $t \in \mathcal{S}$. Report $(\eta, t)$ at $t$ yields maximal continuation reputation to the expert with information $\eta \in\{G, B\}$, so is trivially optimal at the time when he receives his private signal. The uninformed expert's preference for staying silent at any $t$ is at least as high as that of the quack (due to the option value of receiving news in the future and obtaining the maximal continuation payoff). Therefore, it is enough to verify that the quack prefers to stay silent at every point of the support. Since after any report the reputation jumps to $\bar{w}:=\lim _{x \rightarrow+\infty} w(x)$ and to $\bar{w}^{c}:=\lim _{x \rightarrow+\infty} w^{c}(x)$ after state is revealed, in case of no report it must decrease, as $b_{t}$ is a martingale. Therefore, $W_{t_{1}}^{Q}(m, t)$ is maximized at $t=t_{1}$ (and any $m$ ). Report $\left(G, t_{1}\right)$ and report $\left(B, t_{1}\right)$ yield, respectively,

$$
\begin{aligned}
& W_{t_{1}}^{Q}\left(G, t_{1}\right)=\left(T-t_{1}\right) \cdot \bar{w}+p_{0} \cdot \bar{w}^{c}, \\
& W_{t_{1}}^{Q}\left(B, t_{1}\right)=\left(T-t_{1}\right) \cdot \bar{w}+\left(1-p_{0}\right) \cdot \bar{w}^{c} .
\end{aligned}
$$

We have assumed $p_{0} \geqslant \frac{1}{2}$, and therefore $W_{t_{1}}^{Q}\left(G, t_{1}\right) \geqslant W_{t_{1}}^{Q}\left(B, t_{1}\right)$. Using (4), (13), and (14), one can calculate the flow payoff from staying silent until $t$, which equals $w\left(\beta_{0}(1-F(t))\right)$. Therefore, value from not making a report until the last point of $\mathcal{S}$, as evaluated at $t_{1}$, equals

$$
W_{t_{1}}^{Q}(\varnothing)=\sum_{k=1}^{k=|\mathcal{S}|}\left(t_{k+1}-t_{k}\right) \cdot w\left(\beta_{0}\left(1-F\left(t_{k}\right)\right)\right)+(T-\bar{t}) \cdot w\left(\beta_{0}(1-F(\bar{t}))\right)+w^{c}\left(\beta_{0}(1-F(\bar{t}))\right) .
$$

Staying silent is optimal if and only if $W_{t_{1}}^{Q}\left(G, t_{1}\right) \leqslant W_{t_{1}}^{Q}(\varnothing)$. This requires that both $\bar{w}$ and $\bar{w}^{c}$ to be finite, while if $w(\cdot)$ or $w^{c}(\cdot)$ is strictly increasing and convex, it must be that $\bar{w}=+\infty$. 